\title{
Interoperability in Collaborative Networks: Independent and industry-specific initiatives - The case of the footwear industry
}

\author{
Claudia-Melania Chituc $^{\mathrm{a}, \mathrm{b}, *}$, César Toscano $^{\mathrm{b}}$, Americo Azevedo ${ }^{\mathrm{a}, \mathrm{b}}$ \\ ${ }^{a}$ Electrical and Computer Engineering Department, Faculty of Engineering of the University of Porto, Portugal \\ b INESC Porto, Portugal
}

\section{A R T I C L E I N F O}

\section{Article history:}

Accepted 1 December 2007

Available online 19 May 2008

\section{Keywords:}

Interoperability

Collaborative Networks

The footwear industry

\begin{abstract}
A B S T R A C T
The development of information and communication technologies and turbulent market conditions have forced enterprises to adapt their way of undertaking business, from traditional practices to e-business. In this context, achieving seamless interoperability among heterogeneous organizations becomes a critical issue.

This article has two main objectives:

(1) to present a review of the most relevant approaches aiming at achieving seamless interoperability in a collaborative networked environment. In this sense, major interoperability issues (e.g., interoperability requirements, technologies) are discussed, and the most significant independent (e.g., ebXML) and industry-specific initiatives (e.g., RosettaNet, papiNet, TexWeave) are analyzed;

(2) to introduce a recently developed two-layered operational infrastructure aiming at attaining seamless interoperability in the footwear industry (the up-stream segment), based on the requirements identified for this sector in a recent industry survey conducted at European level. Other relevant initiatives targeting this sector are also analyzed, emphasizing the innovative aspects and advantages of this approach.
\end{abstract}

(c) 2008 Elsevier B.V. All rights reserved.

\section{Introduction}

Economic factors, developments of information and communication technologies (ICTs), the adoption of the Internet, and turbulent market conditions have forced small and medium size enterprises (SMEs) to adapt their way of undertaking business, from traditional practices to e-business. In this context, new forms of collaboration have emerged, such as Collaborative Networks (CNs) [1] or virtual enterprises (VEs).

In a collaborative networked environment (CNE), the integration and interoperability enhance the competitive advantages of the CNs and their member organizations. In this context, they become critical goals towards achieving their business objectives in a time, quality and cost effective manner.

Major obstacles faced by SMEs as they engage in e-business are presented in [2]: storage and management of knowledge, skill,

\footnotetext{
* Corresponding author at: INESC Porto, Campus da FEUP, Rua Dr. Roberto Frias 378, 4200 Porto, Portugal. Tel.: +351 968376958.

E-mail address: cmchituc@fe.up.pt (C.-M. Chituc).
}

entrepreneurship; lack of technological solutions and interoperability; investments/costs; complexity of regulations; shortage of capital.

Despite the variety of tools and infrastructures supporting (or claiming to support) seamless interoperability in a CNE, the scientific community and industry representatives agree this objective is not fully achieved (e.g., $[3,4]$ ), and more work needs to be done.

The area of CNs is a relatively new research area, drawing different disciplines, e.g., computer science, economics, management science. Many researchers and developers whose efforts contribute to and benefit from developments in this area are not fully aware of the work pursued so far. Thus, we hope this review of major approaches aiming at achieving seamless interoperability in a CNE will help developers and researchers to define useful directions.

For the footwear industry, concepts like 'supply chain' or 'value chain' are often used. As emphasized in $[5,6]$, they denote a very static structure. We consider that the use of 'Collaborative Networks' concept is more appropriate, because it portrays better the evolution and adoption of ICTs in this sector. Although the 
footwear industry's behaviour is determined by a dynamic seasondriven product demand, its production chain follows a rigid and steady typology. Most relationships, at different chain levels, are quite stable and immutable. This situation presents several drawbacks concerning capacity offer, and prevents companies to exploit new business relationships.

Thus, the approach of $\mathrm{CN}$ paradigm for this sector overcomes these constraints, allowing an easy integration of SMEs or CNs, and the adaptation of the production chain to new business opportunities determined by instant market demands or technological innovations developed on their products and services.

This article has two main objectives:

(1) to present a review of the most relevant approaches aiming at achieving seamless interoperability in a CNE; in this sense, major interoperability issues are discussed (e.g., interoperability requirements, semantic technologies), and the most significant independent (e.g., ebXML) and industry-specific initiatives (e.g., RosettaNet, papiNet, TexWeave) are analyzed;

(2) to introduce a recently developed two-layered operational infrastructure aiming at attaining seamless interoperability in the footwear industry up-stream segment, based on the specific requirements of this sector, which have been identified in a recent industry survey conducted at European level. Other initiatives are also analyzed, emphasizing the innovative aspects and advantages of this approach.

This article has four main contributions. Firstly, it provides an up-to-date review of the most relevant technologies, and independent and industry-specific initiatives aiming at achieving seamless interoperability in a CNE. Secondly, it offers an up-todate insight of the most relevant initiatives targeting the footwear sector, related to business infrastructures. Thirdly, it presents a recently developed two-layered approach towards achieving interoperability in the footwear industry, with emphasis on the up-stream segment, which allows a more integrated and smooth collaboration among its actors (e.g., producer, supplier, sub-contractor). Fourthly, by comparing the initiatives described, it provides recommendations towards improving further industrial interoperability deployments, offering a unique niche for researchers, practitioners and developers to make significant contributions.

The rest of the article is organized as follows. The next section briefly introduces the concept of interoperability in a CNE. The third section provides a review of the most relevant approaches aiming at achieving seamless interoperability; in this sense, the most significant independent (e.g., ebXML) and industry-specific initiatives (e.g., RosettaNet, papiNet, TexWeave) are analyzed and compared. Section 4 portrays the main characteristics of the footwear industry and describes a recently developed two-layered operational infrastructure. An illustrative example of Order
Dispatch Business Process is also described. The paper concludes with a section addressing the needs for future research.

\section{Interoperability in a collaborative networked environment}

This section introduces $\mathrm{CN}$ concept and provides an overview of the main issues related to interoperability in a CNE.

\subsection{Collaborative Networks: a brief introduction}

CNs represent a collection of heterogeneous organizations with different competences, but symbiotic interests that join, efficiently combine the most suitable set of skills and resources for a period of time in order to achieve a common objective, and make use of ICT to coordinate, and support their activities [7]. The term $\mathrm{CN}$ is used in this article, in a broad sense, for other emerging business collaborative forms with similar proprieties, such as VEs. The main differences between CNs and VEs, and a CNE and a Virtual Breeding Environment (VBE) have been pointed out in [8] (e.g., the concepts of CNs and CNE tackle economic aspects, while VE and VBE concepts focus mainly on technical issues).

A CN includes geographically distributed organizations, with different cultures, working methods or supporting technologies. Although CN partners aim at achieving a common business goal by following a common strategy, each member organization has its own goals and strategy, which makes CN coordination, management and inter-organizational communication assume a critical role [7].

CNs have several advantages, the most relevant ones being summarized in [3]: agility, complementary roles, achieving dimension, competitiveness, resource optimization, and innovation.

Main $\mathrm{CN}$ phases (e.g., opportunity identification, creation, operation, and dissolution) are portrayed in Fig. 1, which illustrates also how organizations (e.g., O3) can be part of one or more CNs, while others (e.g., 05, 06) are not invited din any CN and can even disappear, due to bankruptcy, for example (e.g., 06). After identifying the business opportunities, the organizations set-up $\mathrm{CNs}$ and operate towards achieving certain goals. After reaching its objectives, the $\mathrm{CN}$ disappears (e.g., $\mathrm{CN} 1$ in Fig. 1), and its former member organizations search for new business opportunities.

$\mathrm{CNs}$ formation and operation, and their success, depend on some base commonality among its members, such as common goals, common or interoperable ICT infrastructures and supporting services, real-time information sharing and flow among $\mathrm{CN}$ members, common standards or common views on a number of areas (e.g., describing and orchestrating business process, BP, flows across multiple systems, trust, common system of values and common way to perform BPs), as emphasized in [3]. Adequate reference models, supporting infrastructures, proper managerial and technological alignment of inter- and intra-organization BPs

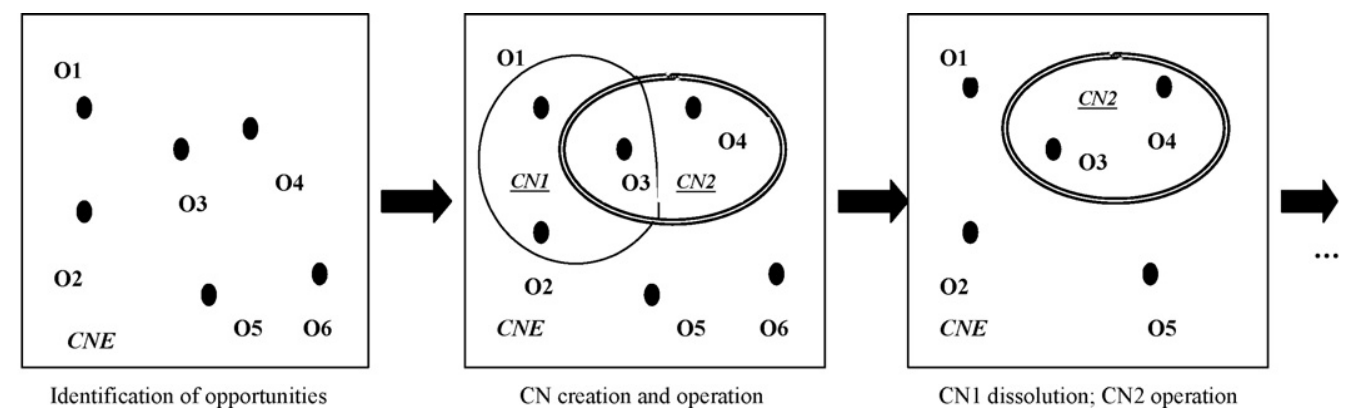

Fig. 1. Main phases of a Collaborative Network. 
are required to achieve these common challenges. A reference model for virtual organization's (VO) planning and launching is available in [9]. A methodology for business model definition of CNs based on an ontological approach is presented in [10].

Although an attractive option to undertake business, since they offer a number of advantages, the CNs face several difficulties, most obvious one being of technological nature, and referring mainly to issues such as inter- and intra-enterprise integration or interoperability. Several inhibitors and challenges for the adaptation of VE concept have been identified in [11], and they refer to: clear organization, adequate workforce, finance, communication, loss of competence, development of the partnership, low acceptance, and intellectual property.

Since the effort required to integrate heterogeneous entities is relatively high, several benefits must be attain to justify this effort. The literature on collaboration identifies some motives and benefits associated with manufacturing inter-enterprise collaboration:

- to increase the market share; to increase asset utilization; to enhance customer service (e.g., reduction in lead times, customer complains); to reduce the cost and time of product development; to share costs; to increase the quality of the products; to increase/enhance skills and knowledge; to have a technological gain as participating firm; to achieve economies of scale in production [12];

- to decrease risk of failure of product development [13];

- to reduce inventory - in the face of increasing technological complexity and rapid rate product development and obsolescence $[13,14]$;

- to gain rapid access to markets $[13,15]$;

- to increase flexibility; to attain international presence for SMEs; to cope with changing dynamics; to have an effective knowledge management [11];

- to have access to resources (e.g., skills, knowledge) that are usually well beyond those of a single player; to learn (e.g., to gain knowledge) form other participants in the network [16].

In order to be competitive in a CNE, organizations should adopt a bipolar approach which allows them to fully benefit from the specific competences of each $\mathrm{CN}$ member [16]: to develop a compatible organizational infrastructure allowing $\mathrm{CN}$ member organizations to share their resources, while supporting the operations to be performed in order to achieve the objectives proposed, and to build up adequate management methodologies, assuring high performance of the business activities.

\subsection{Interoperability: definitions, requirements and main obstacles}

Interoperability, in a broad sense, refers to the use of computer-based tools that facilitate coordination of work and information flow across organizational boundaries, focusing mainly on inter-enterprise distributed BPs and flows. It emerged mainly from the need to harmonize the operational heterogeneous networked environment, real information sharing, and the necessity to improve task coordination.

According to [17], interoperability represents the capability of two or more systems or components to exchange information, and to use it.

As illustrated in [4], interoperability solutions consist of both application integration (e.g., the technology solution: message, process, transport and interface) and information integration (e.g., the linguistic, social and philosophical solution, comprising data, context, ontology and interpreter). Integration here refers to the cooperation of processes and applications at the event and message levels, multiple systems becoming one logical unit.
Three main research domains to address interoperability have been identified by [18]: architectures and platforms; enterprise modelling; and enterprise ontologies. These three areas are concerned with representing the inter-networked organization to establish interoperability requirements; defining implementation solutions to achieve interoperability; addressing semantics necessary to assure interoperability.

A holistic approach (e.g., technological, semantic, business challenges) on CNs' interoperability and business alignment is available in [16]. Major directions requiring further development are summarized in [3], e.g., CN formalization, conceptual development and semantic integration (namely concerning the formal description of the domain or ontology). A roadmap of enablers and technologies supporting interoperability in a collaborative networked environment is available in [1]. An overview of the most relevant elements for VE infrastructures can be found in [3].

Main requirements for interoperability in a CNE are presented in [19]. They refer mainly to technical (e.g., standards, implementation guidelines profiles), business (e.g., business agreements, working methods) and policy (e.g., compliance and governance) interoperability requirements.

Aiming at attaining seamless interoperability in business collaborative environments, several tools and infrastructures have been developed. However, the goal of achieving collaborative environments of full interoperable heterogeneous entities (e.g., people, applications) is still not fully achieved (e.g., [3,4]). Causes are diverse (e.g., inadequate implementation, poorly managed risks and requirements, human errors). This is also due to the fact that research in the area of integration and interoperability is focusing mainly on technological, physical and syntactic aspects. Recent research approaches on semantic issues (e.g., [20,21]) illustrate its potential and benefits for seamless connectivity. Interoperability, in this sense, refers to solutions (e.g., infrastructures, applications), which assure the exchange of minimal meaningful context-driven data and information between autonomous and heterogeneous systems.

Following the gap template proposed in IDEAS project for interoperability between enterprise models [22] and the roadmap on interoperability in enterprise modelling available in [23], the authors have elaborated a gap analysis on inter-enterprise interoperability, which is portrayed in Table 1.

Concerning the as-is situation, there are available many operational infrastructures, standards (e.g., B2B standards, industry-specific standards, frameworks (e.g., ebXML), technologies (e.g., semantic technologies, agents), reference models (e.g., PERA, GERAM) addressing inter-enterprise and cross-industry interoperability (which are presented in Section 3). However, interenterprise and cross-industry interoperability is very low. Furthermore, from inter-enterprise collaboration point of view (e.g., CNs), enterprises should communicate in a standardized or unified way, so that business partners can easily communicate (e.g., interpret the messages exchanged) and choose collaboration partners.

The stat-of-the-art analysis showed that there are no available tools that fully support inter-enterprise and cross-industry interoperability, except for ones that can be used in a very limited scope (e.g., STEP to exchange product information).

The authors have identified four main views on interoperability, towards seamless interoperability in a CNE. They concern: technical aspects (e.g., messaging infrastructure), business/economic issues (e.g., strategic, operational and economic aspects, $\mathrm{CN}$ performance assessment) information (e.g., information sharing and retrieval, messages exchanged) and semantic aspects (e.g., common dictionary, common set of business documents). We argue all these aspects should be tackled by an interoperability 
Table 1

Gap analysis on inter-enterprise interoperability

\begin{tabular}{|c|c|}
\hline \multicolumn{2}{|l|}{ Gap analysis template } \\
\hline \multicolumn{2}{|l|}{ Background } \\
\hline As-is situation & No full inter-enterprise interoperability \\
\hline Desired situation & $\begin{array}{l}\text { Two enterprises can inter-operate (e.g., exchange messages, share knowledge) independent of the languages and tools used, } \\
\text { with a high level of automation (e.g., BPs, collaboration agreements) in a time, quality, and cost effective manner. }\end{array}$ \\
\hline Problem space & Inter-enterprise seamless interoperability, cross-industry seamless interoperability \\
\hline \multicolumn{2}{|l|}{ State-of-the-art } \\
\hline Existing research & $\begin{array}{l}\text { Research projects (e.g., ATHENA), modelling languages (e.g, UMEL), ontology development, reference models (e.g., GERAM), } \\
\text { standards, frameworks (e.g., ebXML), etc. }\end{array}$ \\
\hline Existing technology & $\begin{array}{l}\text { Limited translation and transformation tools, limited ICT platforms/operational infrastructures providing capabilities such as: } \\
\text { multi-level support for interoperability, security, reconfiguration, recovery mechanisms. }\end{array}$ \\
\hline Existing standard & $\begin{array}{l}\text { B2B standards, independent and industry-specific initiatives, standards for automatic exchange of model information (e.g., PSL), } \\
\text { UN/CEFACT UMM, reference models, architectures and frameworks, grid technology, semantic Web, etc. }\end{array}$ \\
\hline \multicolumn{2}{|l|}{ Gap analysis } \\
\hline Research gap & $\begin{array}{l}\text { Lack of formal modelling and general accepted formal definitions. } \\
\text { Lack of mapping between different formalisms. } \\
\text { Lack of research in the area of business/economic and semantic interoperability. }\end{array}$ \\
\hline Technology gap & $\begin{array}{l}\text { No tools and operational infrastructures supporting seamless interoperability are currently available. } \\
\text { Lack of configurable interfaces to support inter-enterprise communication. }\end{array}$ \\
\hline Standard gap & $\begin{array}{l}\text { Standards supporting cross-industry interoperability; standards acceptance } \\
\text { Harmonization of terms and concepts for inter- and cross-industry communication. } \\
\text { Interface standards for inter-enterprise communication. }\end{array}$ \\
\hline
\end{tabular}

framework, and this follows the approach of two EU funded projects: ATHENA (www.athena-ip.org) and IDEAS.

This vision on seamless interoperability relays on a set of attributes, such as: reliability; time (e.g., to set-up or reconfigurate a $\mathrm{CN}$ ); cost (e.g., to set-up or operate a $\mathrm{CN}$ ); openness or extensibility (e.g., the use of open-source software); transparency (e.g., of messages exchanged); scalability, traceability (e.g., of business processes, business documents exchanged), and other non-functional properties, e.g., security. Their selection represents the result of an in depth literature review, analysis of available frameworks, operational infrastructures, and independent and industry-specific initiatives, and the experience gained by participation in national and European R\&D projects in this field.

\subsection{Reference models, standards, frameworks and technologies supporting enterprise interoperability}

This sub-section concisely presents some integration reference models, frameworks, standards referring to B2B domain, and relevant infrastructures and technologies supporting interoperability in a networked environment. According to [24], a reference model represents a (partial) model which can be used as a basis for particular model developments or for the evaluation of particular models. The term framework refers to a collection of elements (e.g., methods, tools) put together for a certain purpose relevant for a given community. Standards can be considered objects (e.g., hardware, software, processes), which are accepted and shared within a community [25].

\subsubsection{Reference models, architectures and frameworks}

SCOR (Supply Chain Operations Reference Model, www.supplychain.org) is a process reference model developed by the SupplyChain Council as cross-industry standard for supply-chain management used to describe, measure, and evaluate supplychain configurations. SCOR model is organized around five primary management processes: plan, source, make, deliver and return. It is composed by a hierarchic architecture of four level details: top level (process types), configuration level (process categories), process element level (decompose processes), and implementation level (decompose process elements).

Workflow Reference Model [30] provides the general architectural framework that identifies interfaces and covers broadly five areas of functionality between a Workflow Management System (WfMS) and its environment: process definitions import and export; interaction with client applications and work-list handler software; software tools or applications invocation; interoperability between different WfMSs; administration and monitoring functions.

PERA (Purdue Enterprise Reference Architecture) provides the reference model of physical and informational interactions in enterprises [26]. An extension of the model for distributed enterprises environment (e.g., VE) is presented in [27].

GERAM (Generalized Enterprise Reference Architecture and Methodology) [28] defines a tool-kit of concepts for designing and maintaining enterprises for their entire life history. GERAM refers to the methods, models and tools, which are needed to build and maintain the integrated enterprise, a single enterprise or a network of enterprises. It encapsulates and orders previous architectures (e.g., CIMOSA, PERA, GRAI/GIM), providing an overall structure to use those methods and modelling techniques. GERAM is not a reference architecture; it is aimed at organizing enterprises' existing integration knowledge.

The Service Oriented Architecture (SOA) has been defined by the Organization for the Advancement of Structured Information Standards (OASIS, www.oasis-open.org) as a paradigm for organizing distributed capabilities, which may be under the control of different ownership domains. It provides means to reorganize applications interacting services.

Zachman's Framework for enterprise architecture [29] describes a holistic model of an enterprise information infrastructure from six perspectives: planner, owner, designer, builder, subcontractor, and working system. Its focus is to ensure that all aspects of an enterprise are well organized and exhibit clear relationships that will ensure a complete system regardless of the order in which they are established.

\subsubsection{Business-to-business integration standards}

In a broad sense, the term B2B integration refers to electronic message exchange among trading partners. It includes issues such as product catalogues, classification systems, B2B protocol standards, synchronous/asynchronous communication, or beckend integration. According to [31], B2B integration is the enabling technology and the necessary infrastructure to perform different operations: e.g., automated supply chain integration, to send XML- 
formatted messages over the Internet, to send messages in a Peerto-Peer (P2P) pattern to trading partners.

According to [32], B2B standards' scope can be roughly separated in three categories, as follows: catalogue systems and classification standards, e.g., eCX (Electronic Catalog XML, www.ecX-xml.org), OCP (Open Catalog Protocol, www.martsoft.com/ocp); Document exchange, e.g., EDI (Electronic Data Interchange), XML (eXtensible Markup Language, www.xml.org), xCBL (XML Common Business Library www.xcbl.org), cXML commerce XML, www.cxml.org; collaboration, e.g., ebXML (Electronic Business XML, www.ebxml.org); business processes (BPs), e.g., BPEL4WS (BP Execution Language for Web Services), UML (Unified Modelling Language, www.uml.org).

Other relevant developments for enterprise integration are: $\mathrm{OBI}$ (Open Buying on the Internet), Bolero.net, eCo framework, BTP (Business Transaction Protocol), XAML (Transaction Authority Markup Language).

\subsubsection{Infrastructures and technologies}

An overview of the current approaches and trends towards the establishment of flexible and configurable infrastructures for a VE is presented in [3], where the supporting emerging technologies are grouped as fallows: open inter-operable underlying network protocols (e.g., TCP/IP); open distributed object-oriented middleware services (e.g., J2EE Framework); information/object exchange mechanisms and tools (e.g., XML, ebXML, WSDL); standardized modelling of business components, processes and objects (e.g., EJBs); BP modelling tools and languages (e.g., UEML, PSL); open and standard BP automation and workflow management system (e.g., XML-WfMC standards); standard interfacing to federated multidatabases (e.g., ODBC); intelligent mobile agents; open and standard distributed messaging middleware systems (e.g., JMS); XML-based e-commerce protocols (e.g., RosettaNet); Web integration technologies (e.g., Servlets, JSP, MS-ASP, XSL). Concerning infrastructures supporting VEs, two of the main approaches (from the software engineering perspective) are presented in [3]: (a) transaction-oriented layer (TOL) based frameworks. TOL infrastructures add a cooperation layer to the existing IT platforms of the enterprises, and inter-enterprise cooperation is then performed via the interaction (transaction-oriented) through these layers; (b) agent-based infrastructures, where enterprises are represented as agents, and the inter-enterprise cooperation as interactions in a distributed multi-agent system.

\subsubsection{Major independent and industry-specific initiatives}

Few studies focus on industry-specific initiatives. The role of industry-based standards development organizations for interorganizational system standard diffusion is discussed in [33,34]. The authors analyse the practices used to develop and deploy inter-organization standards throughout an industrial group, discriminating factors and antecedent conditions towards their implementation and diffusion. However, this study is focusing on the process of diffusion of inter-organization industry standard solutions, and diffusion measures. The study does not tackle interoperability aspects, nor their limitations or challenges.

The following paragraphs briefly present ebXML as major independent approach, and relevant industry-specific initiatives. The decision to analyze these initiatives relays in their capabilities, adoption status and potential in supporting interoperability in a CNE.

2.3.4.1. ebXML. ebXML (Electronic Business XML, www.ebxml.org) provides a standard method to exchange business messages, conduct trading relationships, communicate data in common terms, and define and register BPs. It does not use its own e-business vocabularies (BVs) to describe business documents. Instead, ebXML document types are assembled from core components, which are syntax-neutral descriptions of semantically meaningful business concepts.

ebXML consists in the following elements: a messaging service (ebMS), Registry/Repository, Business Process Specification Schema (BPSS), Collaborative Protocol Profile/Agreement (CPP/ A), and Core Components. At present, the messaging layer has the highest recognition, being adopted by several other initiatives (e.g., papiNet).

An approach to complement ebXML initiative aiming at specifying a methodology to represent syntax-neutral core components in targeted e-BVs is presented in [35]. The framework developed extends ebXML by ontology concepts, in four steps to describe semantically equivalent document types in different eBVs for the same activity in a well-defined BP. However, the first three phases are done manually, and this represents a major limitation of this approach.

ebXML offers a strong conceptual base. However, currently only the messaging service has been implemented and shortly became relatively widely adopted. The implementation of upper layer is quite scarce, efforts being pursued at BPSS and CPP/A levels (e.g., ebBP Editor, www.sourceforge.net). Monteverdi framework (www.monteverdi.com.au) represents another development relying on ebXML.

Opinions on ebXML potential are mixed. While it serves as starting point in some applications [35], there are authors who do not bet much on its future [36]. In our opinion, ebXML represents a powerful basis for inter- and cross-industry developments towards seamless interoperability.

2.3.4.2. RosettaNet. RosettaNet (www.rosettanet.org) develops universal standards for the high-tech and electronics industry global supply chain, and supports their implementation and adoption.

RosettaNet has four main elements: Multiple Messaging Service (MMS) which addresses XML-based business messages exchange and B2B collaboration over horizontal message handling systems; Partner Interface Processes (PIPs) that define BPs between trading partners and PIP Directory; Dictionaries, and RosettaNet Implementation Framework (RNIF) Core Specification is the packaging, routing, and transport of all PIPs messages and business signals. For RosettaNet, business semantics interoperability is achieved through its dictionaries: business and technical.

A major drawback towards achieving integration and interoperability results from the variations in syntax and semantics of PIP action message structures. RosettaNet previously did not establish rules to describe how one supply chain company can modify a PIP via the implementation guidelines specific to that company or by changing the Document Type Definition (DTD), and this generated a large number of varying message semantics for the same PIP, impeding implementation.

A complement to RosettaNet specifications, enabling semantic Web service environment to automated mediation of messages is illustrated in [37].

2.3.4.3. papiNet. papiNet (http://www.papinet.org) is an international paper and forest industry e-business initiative. It is a set of standard electronic business documents which facilitate the flow of information among parties engaged in buying, selling and distribution of paper and forest products.

Its interoperability guidelines are based on ebXML message service specification, being designed to facilitate system-to-system information exchange. Messages have a very uniform structure with common definitions contained in the file that shared amongst all the messages schema files, avoiding in this way semantic 
conflicts. papiNet is offering also a Trading Partner Agreement (TPA) template (currently available only in Word format).

2.3.4.4. CIDX and Chem eStandards. Chemical Industry Data Exchange (CIDX, www.cidx.org) is a non-profit organization aiming at improving the ease, speed, and cost of securely conducting business electronically in the chemical industry. Its focus is on XML-based e-business standards development, called Chem eStandards, which have become the de facto standards for transacting business electronically in the chemical industry.

Chem eStandards comprise BP guidelines, message, envelope and security specifications, and various implementation tools. For long term, it is aimed at developing and maintaining message standards and BP guidelines for common BPs. They are expressed in DTD format, the technology is platform independent, vendorneutral, and are based on open standards. Although the messages are modularized around discrete shared BPs, a single data dictionary is used through CIDX to insure the consistent use and interpretation of the business terms.

Aiming at achieving a more effective and efficient crossindustry trading, CIDX is working with other industry trade associations and standardization bodies (e.g., RosettaNet).

2.3.4.5. Agriculture sector. RAPID (http://www.rapidnet.org) is a not-for-profit organization formed by leading agricultural companies supporting the agriculture community to take advantage of new developments in electronic communication. It makes us of EDI, XML, Bar Coding, Sales Reconciliation Format, unit measure conversion, and endorses Web services interoperability profile concept. Some XML transaction schema files were developed in collaboration with CIDX. It provides a Data Dictionary.

AgXML (www.agxml.org) is an organization of grain and processing companies, and related entities, committed to develop standards for the efficient and effective communication of information electronically through the entire agribusiness supply chain.

2.3.4.6. Petroleum industry. Petroleum Industry Data Exchange's vision (PIDX, http://committees.api.org/business/pidx) is to achieve petroleum industry and enterprise-wide integration of BPs through seamless electronic business communication based on EDI and XML technologies. To tackle semantic interoperability, PIDX is pursuing efforts towards developing a Petroleum Industry Data Dictionary meeting the needs of this industry.

2.3.4.7. Automotive industry. Several initiatives are available for the automotive industry, aiming at developing tools, standards, guidelines, and recommendation for improving the flow of goods, services, product data and business information through the automotive supply chain, such as: the Automotive Industry Action Group (AIAG, www.aiag.org), Odette (www.odette.org), Standards for Technology in Automotive Retail (STAR, www.starstandard.org).

AIAG, STAR, and JAPIA (Japanese Automotive Parts Industry Association), Odette and JAMA (Japanese Automotive Manufacturers Association) jointed their forces to build a global Joint Automotive Data Model (JADM) that will be the foundation for enabling common, consistent business vocabularies at the data level, allowing various business domains to retain their existing infrastructure and BPs. A first version is expected to be released during 2007.

2.3.4.8. TexWeave. TexWeave (Standardization and Interoperability in the Textile Supply Chain Integrated Networks, www.tex weave.org) is a CEN/ISSS standardization initiative aiming at providing an interoperability framework for textile/clothing sector based on standardized electronic document exchange, using XMLSchema and the Internet, and at fostering its adoption in business communities. The results achieved so far comprise business models for fabric and clothing supplying, XML templates for document exchange, and a common dictionary.

\subsection{Semantics and semantic interoperability}

Research on semantics has been pursued on several areas, e.g., computer science, information systems, information retrieval, information extraction, knowledge representation, artificial intelligence, database management, data mining. This section introduces the concepts of semantics and ontologies, and provides an overview on current approaches towards achieving full semantic interoperability.

\subsubsection{Semantics - a brief overview}

Semantics concerns the meaning (e.g., of words, concepts). Semantic interoperability should assure that the meaning of the information exchanged (e.g., business documents, messages) is interpreted in the same way by the communicating systems.

In [20], semantics is organized in three forms: implicit, formal, and powerful. Their roles in enabling some key capabilities related to the Semantic Web are explored. Four kinds of semantics are defined in [38], considering a semantic continuum view: implicit; explicit and informal; explicit and formal for human processing; explicit and formal for machine processing.

An overview of scientific semantic issues is available in [39]: inter-connection semantics (e.g., single semantic image, transformation and consistency between semantic spaces), semanticbased storage and retrieval, semantically rich interfaces, and the evolution of semantics, normalized resource organization, and intelligent clustering and fusing.

Heterogeneity represents an enormous advantage, especially in the case of CNs, since it provides access to resources or technologies beyond an organization's capabilities. However, heterogeneity of knowledge or data format, for example, is a barrier towards systems' seamless connectivity.

Four types of heterogeneity are identified in [40]: paradigm heterogeneity (e.g., two systems express their knowledge using different modelling paradigms); language heterogeneity (e.g., two systems express their knowledge in different representation languages); ontology heterogeneity (e.g., two systems make different ontological assumptions about their domain knowledge), and content heterogeneity (e.g., two systems express different knowledge). The first two types are considered non-semantic heterogeneity, while the last two represent examples of semantic heterogeneity.

In a CNE, heterogeneity generates semantic conflicts among systems. They represent misunderstanding between the content and intended meaning of heterogeneous communicating systems. According to [4], this comes in three forms: confounding conflicts (e.g., information appears to have the same meaning, but does not), scaling conflicts (e.g., different reference systems measure the same value), and naming conflicts (e.g., synonyms and homonyms). Ontologies are being used to describe information and also as a tool to resolve semantic heterogeneity conflicts.

\subsubsection{Ontologies}

An ontology represents a formal and explicit specification of a shared conceptualization [41]. Conceptualization refers to an abstract model identifying relevant concepts. The types of concepts used and their constraints are explicitly defined. An ontology should be machine understandable, capturing consensual knowledge. A survey on ontology creation methodologies is available in [42]. 
Two ontology typologies are presented in [43], according to the level of formality (e.g., informal ontology, terminological ontology, formal ontology), and the level of granularity (e.g., top-level ontology, general ontology, domain ontology, task ontology, application ontology, and meta-ontology). Three different directions on how to employ ontologies (single, multiple, and hybrid topology approach) are presented in [44].

The reason for ontologies being so popular is in large part due to what they promise: a shared and common understanding of some domain that can be communicated across people and computers [45]. Main application areas of ontology technology are presented in [46]: knowledge management, Web commerce, and electronic business. However, representing data in ontologies does not solve semantic conflicts. According to [4], to achieve this objective, ontology has to be connected to real applications with metadata and context descriptions.

\subsubsection{Towards full semantic interoperability}

When heterogeneous entities collaborate, a common frame of reference or at least a common terminology is necessary to assure communication (e.g., machine-to-machine or human-to-machine communication). Misunderstandings between humans and machines or between applications (e.g., business related terms or tasks) or inadequate translation or transformation are obstacles in achieving interoperability.

This section analyses the most relevant approaches aiming at achieving semantic interoperability.

\subsubsection{Standard based approaches: process specification language} . Several initiatives having as starting point Process Specification Language (PSL) have been developed: e.g., [47-50]. ISO 18629 PSL standard aims at creating a neutral, high-level language for specifying processes and the interaction of multiple process-related applications throughout the manufacturing life cycle.

PSL (http://www.mel.nist.gov/psl) is a neutral, standard description of manufacturing processes expressed in Knowledge Interchange Format. The foundation of the process specification language is the PSL ontology, which provides rigorous and unambiguous definitions of concepts necessary for specifying manufacturing processes to enable exchange of process information. It provides translation mechanisms between individual applications and PSL: e.g., syntactic translation, semantic translation to PSL, or from one application to another.

An approach for semantic integration using model-theoretic invariants and PSL is available in [47]. Limitations of this approach concern the incapacity to define first-order invariants, and difficulty to determine the correctness of translation definitions.

Given its properties and structure, PSL represents a powerful interoperability tool for enterprises' information systems, capable of resolving semantic ambiguities. PSL's appeal is that it processes a standard content language for the exchange of processes between heterogeneous applications. It also holds a promise as a content language for agent communication in manufacturing agent-based systems by providing unambiguous concept definitions for the content layer of agent-communication languages [50].

Although the automation of translation has been prototyped, this approach presents some limitations: there is a need to reach critical mass for design and associated software applications, and there is no Web implementation currently available or use of stateof-the-art Web protocol.

2.4.3.2. Semantic technologies. Major near-term projects where semantic technologies can provide key benefits are semantic Web services, intelligent search, and Semantic Grid.
Semantic technologies support the requirements for semantic interoperability by offering a framework for connecting distributed data and describing it in a context sensitive-way. The use of semantic technologies makes it possible to describe the logical nature and context of the information being exchanged, while permitting communicating parties maximum independence. As emphasized in [51], the results are greater transparency and more dynamic communication among communities irrespective of business logic, processes, and workflows. The authors also present a scenario towards full semantic interoperability (based on semantic technologies).

Intelligent search is related to some regard to semantic interoperability. Semantic interoperability techniques can allow queries native to one system to be federated to other non-native systems. This eliminates the need to convert systems to a universal query language.

Several research efforts are now being pursued towards enriching Web services with machine-processable semantics. Web services approach relies on the assumption that every system using them will be "speaking" the exact same language and dialect (e.g., agree to speak about everything in the same way), or they require a translation service to enable systems communication. However, as underlined in [4], this approach will generate similar problems as occurred in the case of EAI. Their main drawback is that they rely strongly on vocabularies. Vocabularies can be significant for specific industries/communities, but it is naïve to believe that a single set of standards will enable businesses everywhere to speak precisely the same vocabulary.

Semantic Web services have the potential to achieve dynamic, scalable and cost-effective marketplaces. The semantic Web services provide access to heterogeneous, distributed information. They address the ontological limitation of the current Web. However, all service descriptions are based on semi-formal natural language descriptions, which require human involvement, generating limited scalability and economy of services. Semantic Web services allow mechanization in service identification, configuration, comparison and combination.

Semantic Web services leverage the Web Ontology Language Service (OWL-S, http://www.w3.org/Submission/OWL-S/) specifications to provide a flexible framework for describing and initiating Web services. The two key features of the Semantic Web approach are: machine processable descriptions, and sharing and accumulation.

Semantic Grid (http://www.semanticgrid.org) is an extension of the current Grid, in which information and services are given welldefined meaning, enabling computers and people to cooperate [52]. It is an Internet-centered interconnection environment that can effectively organize, share, cluster, fuse and manage globally distributed versatile resources based on the interconnection semantics [39].

All these semantic technologies approaches are, at their core, an infrastructure capability, which, combined with other key technologies, bring major steps towards achieving semantic interoperability.

2.4.3.3. Architectures' development. A survey on architectures that may be used to achieve semantic integration and interoperability is available in [53]. The authors have identified the following dimensions of variations: origins of the semantic mappings, mediating ontology, nature and degree of the agreements that exist among the anticipated community of interacting agents. Five architectures that can be used to integrate agents have been identified, considering mappings generator, topology and the degree of agreement: global ontology, manual mapping, interlingua ontologies, community ontology mappings, and ontology 
negotiation. These architectures are not mutually exclusive alternatives; they are the building blocks for a semantically connected network (e.g., of agents, data sources, applications) [53]. All these approaches are in evolving phases, and they can be combined, or complemented with the emerging technologies.

2.4.3.4. Business vocabularies. An industry-specific business vocabulary (BV) contains all the specialized terms and definitions of concepts that a given organization or community uses while undertaking business. Standards have proven to be a relatively slow and difficult way to develop common vocabularies; e.g., it is rather difficult to change them, and they can be easily misused.

Several organizations have developed their own vocabularies. Lacking guiding standards for interoperability, the solutions attained use different data structures and tagging to code the same business concept [35]. Relevant examples of e-BVs are: Commerce One's xCBL (XML Common Business Library, http://www.xcbl.org), Ariba's cXML(CommerceXML, http://www.cxml.org), and industryspecific BVs. An overview of e-BVs is presented in [19].

Several initiatives are under way to define standards for business rules, in particular OMG's Semantics of Business Vocabulary and Business Rules (SVBR). SVBR includes two specialized vocabularies (http://www.w3.org): SVBR 'Vocabulary for Describing Business Vocabularies' (VDBV), dealing with terms and meanings, and SVBR 'Vocabulary for Describing Business Rules', dealing with the specification of the meaning of business rules and builds VDBV.

\subsubsection{UN/CEFACT's modelling methodology. UN/CEFACT (United} Nations Centre for Trade facilitation and Economic Business, http://www.unece.org) modelling methodology (UMM) (http:// www.gfeg.com/tmwg) aims at defining business collaboration models for B2B based on UML. The UMM meta-model describes the business semantics that allows trading partners to capture the details for a specific business scenario using a consistent modelling methodology based on UML.

2.4.3.6. Final remarks. All the above-mentioned approaches represent a step forward towards achieving seamless connectivity in a CNE. However, they all require further research and developments. Table 2 illustrates their major drawbacks, and possible solutions to overcome their limitations.

Among main barriers towards achieving full semantic interoperability identified are: lack of guiding standards for interoper- ability (e.g., in building BVs); lack of an automatic way for mapping, aligning, integrating, merging of ontologies; difficulties to identify and solve ontology mismatches; none of the current integration technologies (e.g., B2B integration, EDI, CORBA, EAI tools) solves the issue of incompatible business semantics; no automated solution to resolve incompatible business and data vocabularies for the above mentioned technologies (e.g., currently these problems are solved by custom code)

A relevant challenge in achieving semantic interoperability refers to getting existing e-business vocabularies to interoperate. Semantic Web clearly involves distributed Resource Description Framework (RDF) stores, and not single standalone solutions with single ontologies. This is, in fact, one of the challenges in the Semantic Grid [54].

Several tools working with RDF data are currently available (e.g., editors, browsers, stores or RDF triple-stores) which can be queried using different languages (e.g., SPARQL Protocol and RDF Query Language). Tools working with vocabularies/ontologies are also available (e.g., to check consistency, aid maintenance). However, such approaches are at an early deployment stage [54], and they are corroborated with other difficulties, such as unfriendly user interfaces.

\section{Operational infrastructure for the footwear industry}

This section portrays the main particularities of the footwear industry, its requirements for seamless interoperability, and the main outcomes of relevant initiatives related to business infrastructure. A two-layered operational infrastructure recently developed within the scope of CEC-made-shoe EU Integrated Project (http://www.cec-made-shoe.com) aimed at achieving seamless interoperability in this sector is described, with emphasis on the up-stream segment.

\subsection{The footwear industry: a brief presentation}

The footwear industry has several particularities; the most relevant ones have been summarized in $[5,6,55]$. This sector is characterized by a season-driven product lifecycle. Marketing campaigns are linked to exhibitions and fairs tied to two typical season collections, for summer and winter. Its main product lifecycle activities refer to collection creation, sample production, sale campaign, production, finished product stock, shop sales monitoring, and re-orders.

Table 2

Approaches aiming at semantic interoperability in CNs: major drawbacks and possible solutions

\begin{tabular}{|c|c|c|}
\hline Approach & Major drawbacks & Possible solutions/further research direction \\
\hline Semantic Web services & - Rely strongly on vocabularies & - Combination with other approaches (e.g., Grid technologies) \\
\hline Vocabularies & $\begin{array}{l}\text { - Difficult to make everybody use the same vocabulary } \\
\text { - No automatic solution to resolve incompatible business data } \\
\text { - Inter- and cross-industry difficulties in data interpretation }\end{array}$ & $\begin{array}{l}\text { - Vocabularies for industry-specific initiatives } \\
\text { - Development of tools for the automatic interpretation } \\
\text { of the meaning }\end{array}$ \\
\hline Ontologies & - Do not solve semantic conflicts. & $\begin{array}{l}\text { - Further development. } \\
\text { - Connection to real applications }\end{array}$ \\
\hline Semantic Grid & - Evolving phase & $\begin{array}{l}\text { - Further development, absorbing other technologies } \\
\text { (e.g., semantic Web services, communication platforms) }\end{array}$ \\
\hline Languages & $\begin{array}{l}\text { - Not enough critical mass. } \\
\text { - No Web implementation platforms available. }\end{array}$ & - Further development and connection to real applications \\
\hline Architectures & - Are still in an evolving phase. & -Further development \\
\hline Industry-specific approaches & $\begin{array}{l}\text { - Misunderstandings for cross-industry communication } \\
\text { - Incipient phase }\end{array}$ & $\begin{array}{l}\text { - Joint research projects and active collaboration among } \\
\text { different industry communities } \\
\text { - Further developments }\end{array}$ \\
\hline
\end{tabular}


For this sector, a make-to-stock production approach is suitable only for classic, non-fashionable shoes, which have stable and high volumes of order. Most other models follow a make-to-order approach. This determines lower and more frequent production changes, and higher costs. Most of the stock handling is thus related to products already ordered.

The footwear sector is characterized by a centralized procurement: shoe producers often contract different companies for labour-intensive production phases. However, even if these production phases are delegated, tanneries' leather procurement is usually carried out by the producer, in order to assure constant leather treatments when the same model is cut and stitched by different sub-contracted companies.

A trend commonly identified is the increase of the number of models offered, with very low volumes. In this way, traditional shoe producers are forced to focus on high-quality, and highfashion shoes, which are available in very small quantities, in a make-to-order approach.

The most relevant actors of the footwear industry (up-stream segment) are: the Producer (P), which represents the SME responsible for the production of the final product; Sub-contracted company ( $\mathrm{SbC})$, which is the SME sub-contracted by P to manufacture parts of a shoe or even the entire shoe; Supply company (SppC), which provides raw material and/or shoe components to $\mathrm{P}$ and/or SbC.

The main roles performed by the first three main actors are: buyer, seller, and delivery recipient; e.g., in $\mathrm{P}-\mathrm{SbC}$ relationship, $\mathrm{P}$ is the actor performing the role of buyer and delivery recipient, and the $\mathrm{SbC}$ is the seller.

\subsection{Main findings}

The need for an operational infrastructure addressing the requirements of the actors involved in the footwear industry and assuring seamless interoperability in this sector have been the main reasons that have justified CEC-made-shoe EU Integrated Project. A survey conducted among different SMEs performing different roles (e.g., Ps, SppCs, and SbCs) validated these needs.

The most relevant results can be summarized as follows: SppCs SMEs mainly do business with SMEs actin $\mathrm{g}$ as $\mathrm{P}$; no relationship with the retailers has been reported; $85 \%$ of production is customized production; closer integration/communication with $P$ has been indicated as having a big impact on SppC profits; closer integration with own suppliers and end-product sellers have been reported as having a low impact on SppCs' revenues. Just one third of the SppCs participating in this inquiry offer their products in an Internet-based B2B system. General cooperation between SppCs and $\mathrm{P}$, and limitation of Ps' clients systems have been indicated as main bottlenecks in this business collaborative environment. An increased productivity and the reduction of the overall costs; enhanced customer service and responsiveness, faster time to market, reduction in total order fulfilment times, increase in market share, expand to new markets have been indicated as the most important objectives to be achieved with the use of ICTs in this sector. These results are also pointed out in [56].

As emphasized in [6], CNs' paradigm overcomes these constraints. It eases the integration of new links among SMEs in the footwear industry, and production chains' dynamic adjustment to new business opportunities determined by instant market demands.

The results gathered allowed a better understanding of the requirements and characteristics of this sector. They have provided a strong base in designing and implementing an interoperable operational infrastructure. Very important is the fact that the SMEs interviewed foresee the use of Internet and XML-based technologies to perform business.

\subsection{Relevant initiatives}

The recently developed two-layered operational infrastructure implemented represents an initiative beyond relevant initiatives focusing on this sector, such as:

EFNET project (European Footwear Network for Electronic Trading, http://www.efnetwork.org) aimed at building a common communication infrastructure ensuring information distribution along the Footwear Supply Chain. EFNET1 has identified ecommerce initiatives, which have reached the stage of operational use, developed on national basis. EFNET2 proposal covered data exchanged in the reselling phase, considering the standardization work of ebXML. EFNET 3 proposal is on business data exchanged in the first ring of the footwear chain.

ShoeML (Shoe Markup Language) represents an XML-based language used to store footwear design data, related to EFNET 2 and EFNET 3 schemas. It is a framework of XML schemas with a general structure modelled after the needs of design data storage and exchange.

SHOENET project (http://www.shoenet.info) had two main outcome: (1) a messaging platform able to integrate in the Internet existing corporate applications (e.g., ERP) of different SMEs participating in the shoe value chain, allowing secure business documents exchange and the traceability of the operations performed; (2) a set of 17 XML-based business documents (BDocs) especially designed for this sector.

\subsection{CEC-made-shoe EU integrated project}

\subsubsection{Introduction}

The on-going CEC-made-shoe EU Integrated Project (http:// www.cec-made-shoe.com) aims at achieving seamless interoperability in the footwear sector. It continues the above mentioned initiatives, making use of SHOENET messaging platform and the set of 17 XML-based SHOENET BDocs defined for this industry. One of its main expected outcomes is an operational platforms, called the Strategic Collaborative Network (SCN), covering all BPs involved (from collaborative design to final product delivery). The SCN has two levels of business integration (Fig. 2): (a) a secure system for BDocs reception and transmission; (b) the coordination and enactment of workfloworiented activities, which ensures BP choreography [57]. The business model developed is being tested for several business integration scenarios.

The common set of 17 XML-based SHOENET BDocs assures semantic interoperability among footwear SMEs. They represent the only data model defined for this sector.

\subsubsection{Sample business scenarios}

Different BPs and business scenarios (BSs) to be supported by the operational infrastructure have been identified. Based on them, the specifications of the infrastructure design will evolve in order to prepare its implementation and automation.

Four examples of general BSs are briefly presented below:

BS1: The Producer (P) orders raw material and/or components from different Suppliers. The complete shoe manufacturing process is performed by $\mathrm{P}$.

BS2: P orders raw material and shoe components from different Suppliers, and subcontracts SbCs to manufacture (partially or totally) the shoes. Only $\mathrm{P}$ paces orders. The raw material and shoe components are delivered to $\mathrm{P}$ and/or directly to the SbCs involved. Fig. 3 presents a simplified instantiation of this BS. BS3: Variations of the previous scenario, where: (a) SbCs may place orders directly to their own Suppliers; (b) SbCs are 


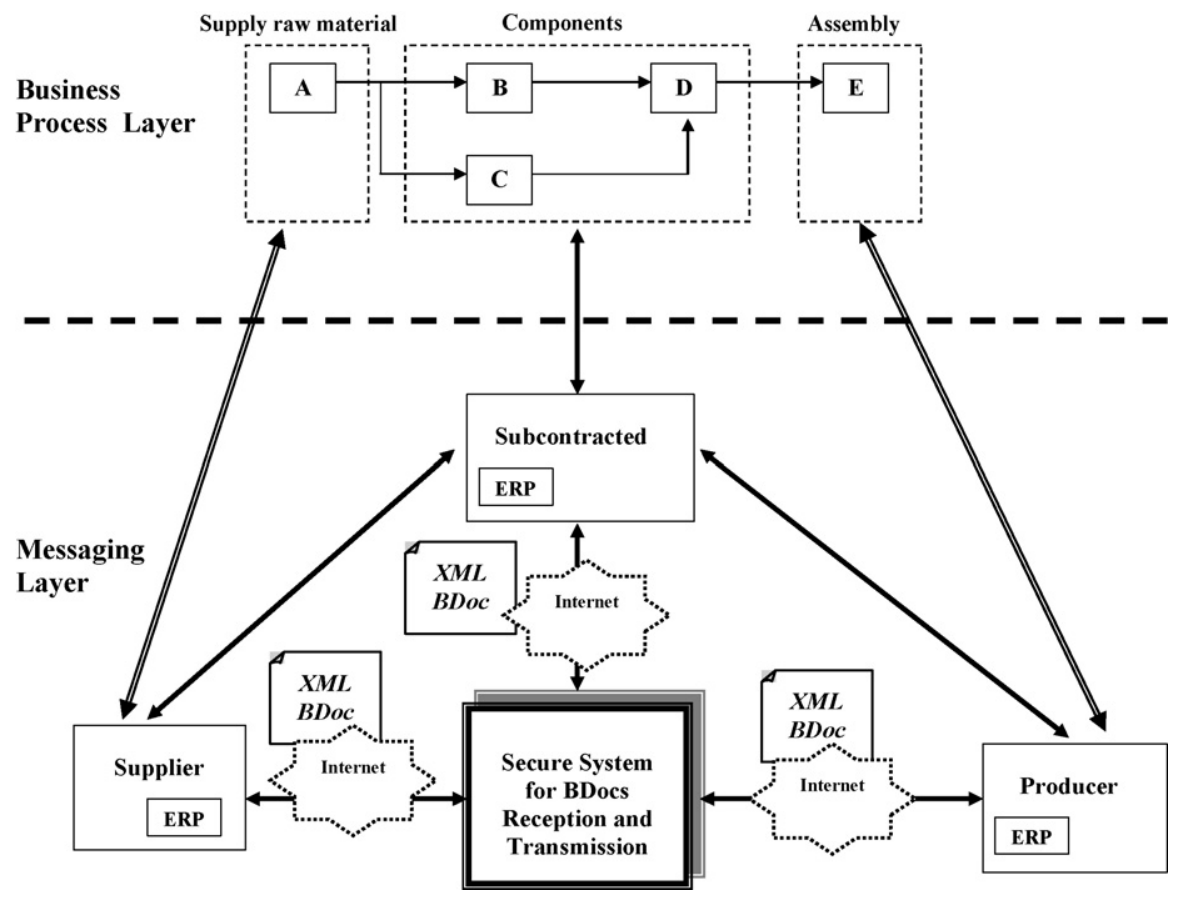

Fig. 2. Two-layer business infrastructure for the footwear industry (up-stream segment).

allowed to place orders directly only to certain Suppliers, previously selected by $P$.

BS4: $P$ sends a forecast of future raw material and/or shoe components needs to a set of SppCs, who, based on the data received, prepare themselves for the best response times. Future purchase orders placed by Ps will refer previous forecast.

\subsubsection{Messaging infrastructure development}

Achieving seamless interoperability in the footwear industry requires a reliable and secure communication infrastrcuture among SMEs. Several messaging platforms and industry-specific initiatives have been analyzed. After an in depth analysis, the research team has decided to adopt SHOENET platform as messaging layer. Main reasons for this decision are: it assures reliable and secure communication among SMEs; this messaging platform has been previously tested and used in SHOENET project; it supports SHOENET BDocs transmission and reception. However, it presents a drawback: it is a proprietary messaging platform, not compliant with ebXML Messaging Specifications.

The BDocs are stored in a repository. The envelops are opened, and external applications (e.g., ERP solutions) may use API to send/ receive $\mathrm{BDocs}$ to/from external partners.

An additional element has been developed: eBiss/eGate system. It assures a reliable and secure transmission of BDocs

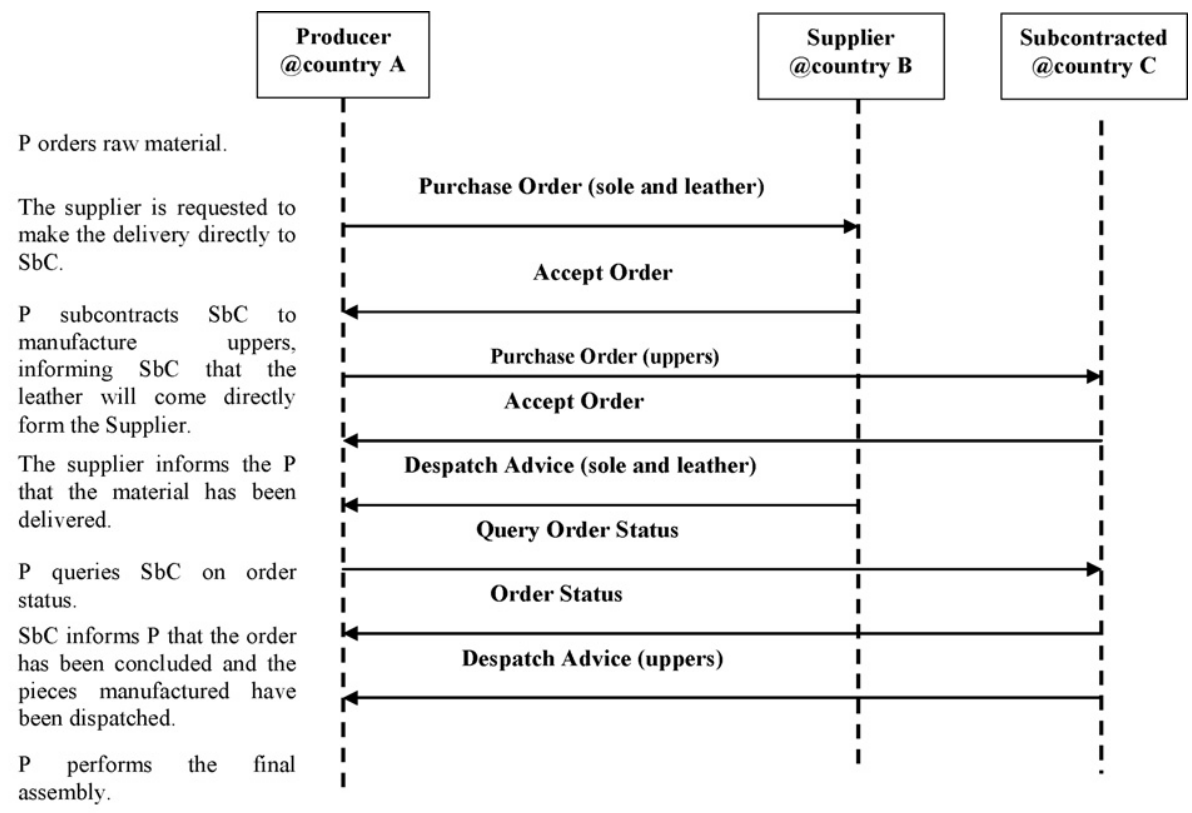

Fig. 3. Simplified instantiation of Business Scenario 2. 


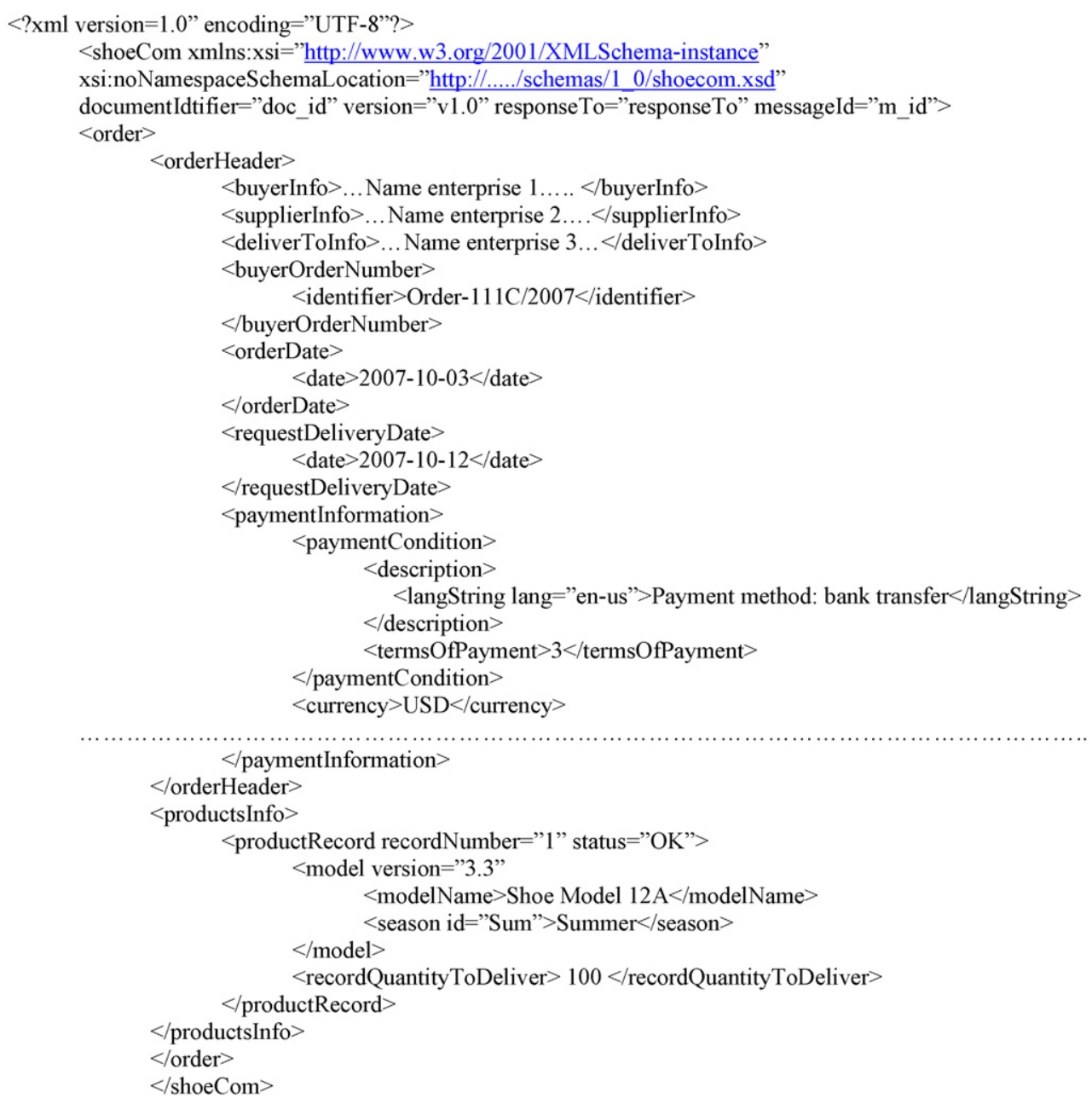

Fig. 4. Excerpt of an Order SHOENET business document.

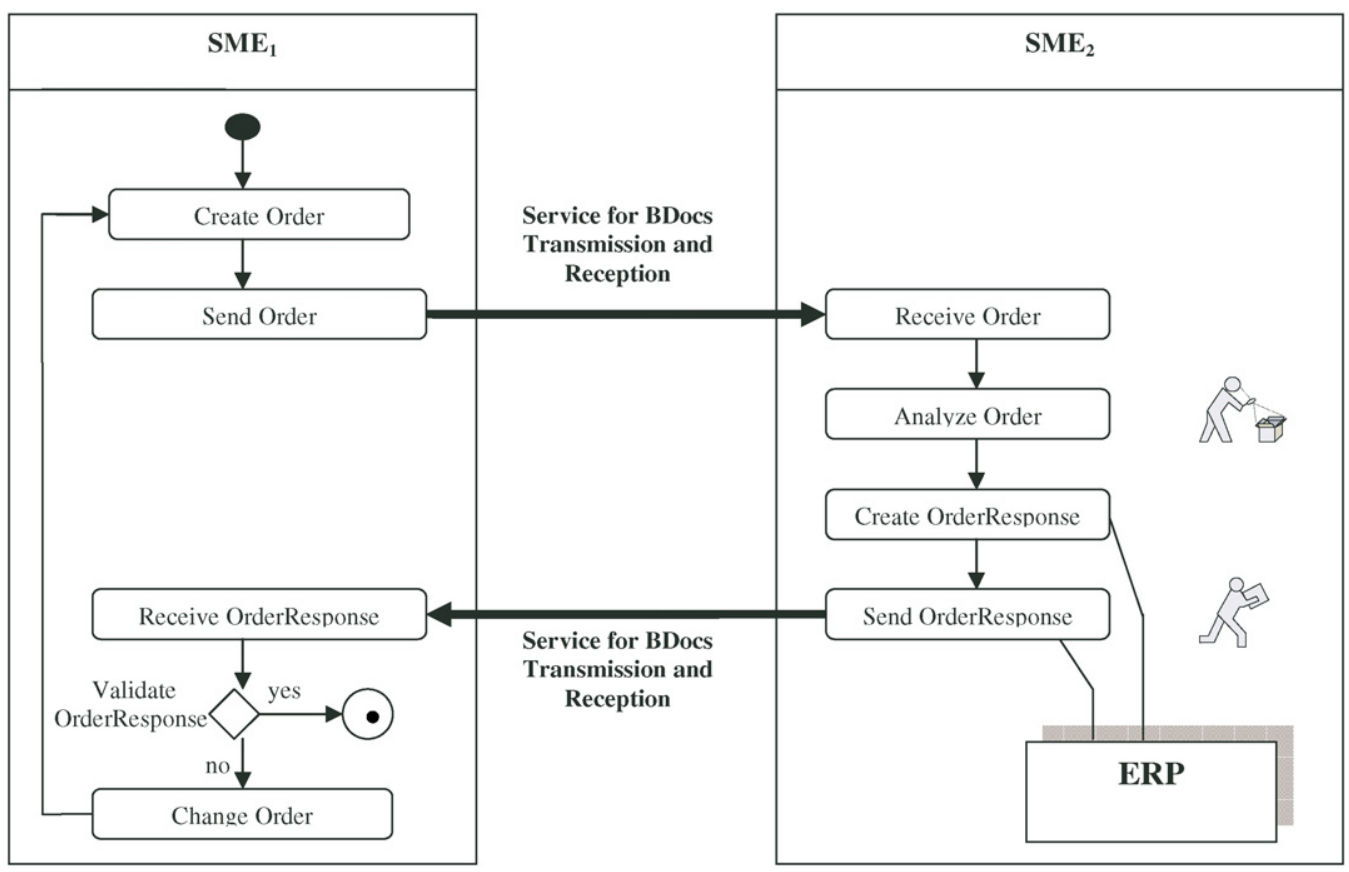

Fig. 5. PICShoe System: An example of an Order Business Process. 


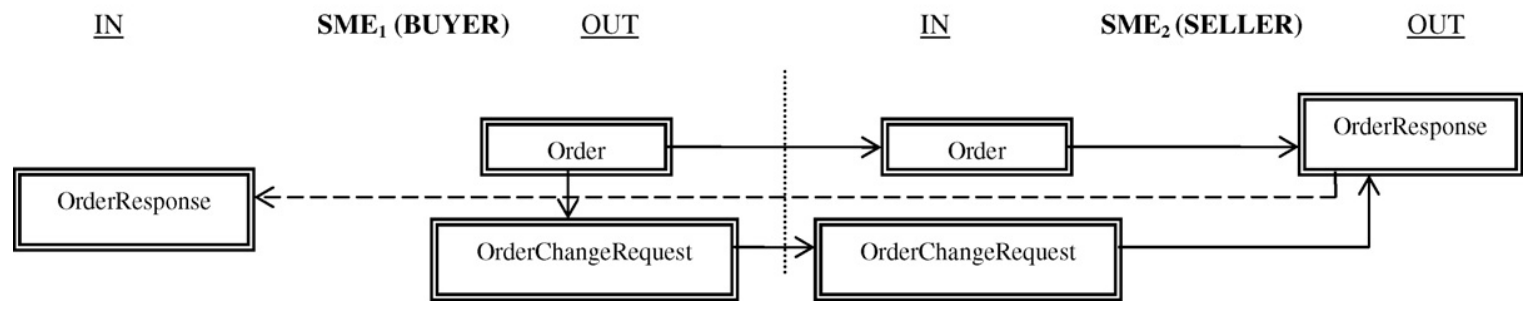

Fig. 6. Example of business documents flow for an Order Business Process.

among the actors, and is responsible for the integration of the SCN with other platforms.

\subsubsection{XML-based business documents}

Aiming at covering most of the different collaborations and relationships among the main actors in the footwear sector, a set of 17 XML-based BDocs has been developed for this sector (see Section 4), which can be grouped in three main categories: a) preorder management (e.g., Quotation, RequestForQuotation BDocs); b) order management (e.g., Order, OrderResponse, OrderChangeRequest BDocs); c) payment (e.g., Invoice, CreditNote BDocs). A sample of an order SHOENET BDoc is illustrated in Fig. 4.

\subsubsection{Business process layer}

The BP layer supports a process-oriented view on the business interactions among different business partners. The boxes $\mathrm{A}, \mathrm{B}, \mathrm{C}$ and D portrayed in Fig. 2 are workflow activities in a certain workflow process. The workflow processes at the different business partners will be integrated. The integration of each company's workflow processes is realized through the invocation of external Web services. Some workflow activities in a workflow process will need to be integrated with third-party applications of another organization (e.g., ERP). These business partners will make available a set of Web services whose invocation will be performed and coordinated by a BP integration and coordination system, which should be able to differentiate human-based from automatic activities, and coordinate their assignment and execution. Each inter-enterprise BP may be specified as two separate workflow processes, which are coordinated or executed by separate workflow engines in each SMEs' environment.

This layer is implemented by a Process Integration and Coordination System, PICShoe, which assures BPs coordination

(a)

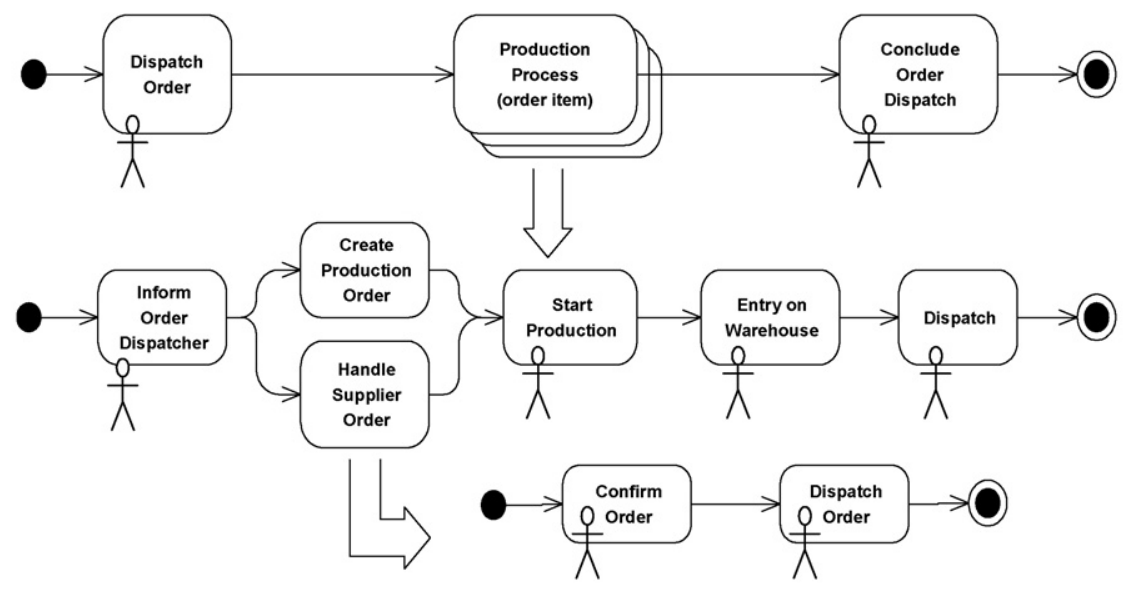

(b)

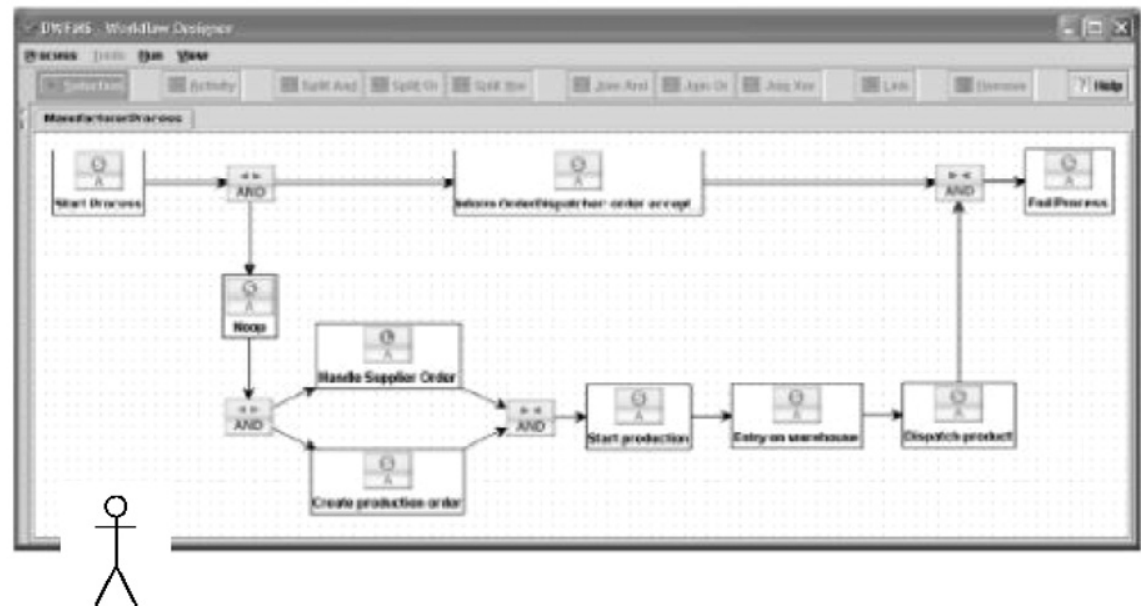

Fig. 7. Order Dispatch BP: (a) conceptual design; (b) workflow process design. 
Table 3

Relevant independent and industry-specific initiatives: a brief overview

\begin{tabular}{|c|c|c|c|c|c|c|}
\hline Initiative & Web site & Start & Focus & Aim & Promoters/supporting bodies & Versioning \\
\hline ebXML & www.ebxml.org & 1999 & SMEs in general & $\begin{array}{l}\text { To provide specifications that enable } \\
\text { enterprises of any size and in any } \\
\text { geographical location to conduct } \\
\text { business over the Internet. }\end{array}$ & OASIS, UN/CEFACT & $\begin{array}{l}\text { Core components technical specifications } \\
\text { of ebXML Technical Framework v2.0.1; } \\
\text { ebBP v2.0.4; Messaging Service v3; } \\
\text { Registry Service v3.0; Registry } \\
\text { Information Model v3.0; ebXMl CPP/A v2 }\end{array}$ \\
\hline RosettaNet & www.rosettanet.org & 1998 & $\begin{array}{l}\text { High-tech and } \\
\text { electronics industry }\end{array}$ & $\begin{array}{l}\text { To develop universal standards for } \\
\text { the high-tech industry global supply } \\
\text { chain, supporting their } \\
\text { implementation and adoption. }\end{array}$ & $\begin{array}{l}\text { Founded in Silicon Valley. } \\
\text { Currently, it has affiliates } \\
\text { in Europe, Asia and Australia. }\end{array}$ & $\begin{array}{l}\text { PIPs, RNIF v02.00.01, MMS ebMS Profile } \\
\text { r11.00.00A, MMS AS/2 Profile r01.00.00A, } \\
\text { RNTD v4.1.1, RNBD v2.1 }\end{array}$ \\
\hline papiNet & www.papinet.org & 2000 & $\begin{array}{l}\text { Paper and forest } \\
\text { products industry }\end{array}$ & $\begin{array}{l}\text { To provide a set of standard electronic } \\
\text { documents that facilitate the flow of } \\
\text { information among parties engaged } \\
\text { in buying, selling and distributing } \\
\text { paper and forest products. }\end{array}$ & $\begin{array}{l}\text { More than } 80 \text { companies from } \\
4 \text { continents (e.g., CEPI, GCA) }\end{array}$ & V2.3.1 \\
\hline Chem e -Standards & www.cidx.org & 1985 & Chemical industry & $\begin{array}{l}\text { To improve the ease, speed and cost } \\
\text { of securely conducting business } \\
\text { electronically in the chemical industry. }\end{array}$ & $\begin{array}{l}\text { Chemical Industry Data } \\
\text { Exchange (CIDX) }\end{array}$ & $\begin{array}{l}\text { Chem eStandards v3.0; CIDX } \\
\text { Documents v4.0; } \\
\text { BP Guidelines v1.0; Implementation } \\
\text { Accelerator v1.2; }\end{array}$ \\
\hline RAPID & www.rapidnet.org & 1995 & Agriculture & $\begin{array}{l}\text { To enable agriculture and related } \\
\text { businesses to achieve the economic } \\
\text { and stewardship benefits of e-business; } \\
\text { to facilitate effective and efficient } \\
\text { implementation of e-business among } \\
\text { its members. }\end{array}$ & Agriculture companies & RAPID v3 \\
\hline AgXML & www.agxml.org & & Grain processing & $\begin{array}{l}\text { To develop standards for efficient and } \\
\text { effective communication throughout the } \\
\text { entire agribusiness supply chain. }\end{array}$ & $\begin{array}{l}\text { Grain and processing } \\
\text { companies, and related entities }\end{array}$ & AgXML v2 \\
\hline PIDX & $\begin{array}{l}\text { http://committees.api.org/ } \\
\text { business/pidx }\end{array}$ & 1986 & Petroleum and oil & $\begin{array}{l}\text { To achieve petroleum industry and } \\
\text { enterprise-wide integration of BPs through } \\
\text { seamless e-business communication. }\end{array}$ & $\begin{array}{l}\text { American Petroleum Institute, } \\
\text { Shell, Total, ChevronTexaco, etc. }\end{array}$ & $\begin{array}{l}\text { PIDX } 3901 \text { (first XML-version } \\
\text { available in 2001) }\end{array}$ \\
\hline JADM & & & Automotive industry & $\begin{array}{l}\text { Provide international standard direction } \\
\text { for developing business content, seamless } \\
\text { interactive business communication for the } \\
\text { automotive industry. }\end{array}$ & $\begin{array}{l}\text { AIAG; AutoDESA; CCAQ; CIECA; } \\
\text { MIC; OAG; STAR, etc. }\end{array}$ & V1 (expected in 2007) \\
\hline TexWeave & www.texweave.org & 2005 & Textile industry & $\begin{array}{l}\text { To provide the textile/clothing sector with } \\
\text { a framework for interoperability based on } \\
\text { standardized electronic document exchange } \\
\text { based on schemas and the Internet, and to } \\
\text { foster its adoption in business communities. }\end{array}$ & EURATEX & XML templates v2 (March 2006) \\
\hline Cec-made-shoe & www.cec-made-shoe.com & 2004 & Footwear industry & $\begin{array}{l}\text { To achieve seamless interoperability in the } \\
\text { footwear industry. }\end{array}$ & EU consortium partners & V1 (expected in 2007) \\
\hline
\end{tabular}


Table 4

CN's interoperability and independent and industry-specific initiatives: drawbacks and challenges

\begin{tabular}{|c|c|c|c|c|}
\hline Initiative & Main elements & Approach towards semantic interoperability & Major drawbacks & Possible solution/challenges \\
\hline$e b X M L$ & $\begin{array}{l}\text { Messaging/transport; Registry/Repository; } \\
\text { business documents; business process } \\
\text { specification schema; collaboration } \\
\text { protocol profile/agreement; core components. }\end{array}$ & $\begin{array}{l}\text { Core components/business documents } \\
\text { (assembled from syntax neutral core } \\
\text { components) }\end{array}$ & $\begin{array}{l}\text { Lack of semantic framework/ } \\
\text { semantic support }\end{array}$ & $\begin{array}{l}\text { Enrich its semantics with the use of } \\
\text { ontologies (e.g., OWL at Registry/Repository } \\
\text { level) or e-vocabulary and development } \\
\text { of a semantic framework }\end{array}$ \\
\hline RosettaNet & $\begin{array}{l}\text { Multiple messaging service ( } 3 \text { predominant } \\
\text { messaging systems: ebMS, Web services, } \\
\text { AS/2), PIPs \& PIP Directory; dictionaries } \\
\text { (business \& technical); RosettaNet } \\
\text { implementation framework. }\end{array}$ & Business dictionary & $\begin{array}{l}\text { Variations in syntax and } \\
\text { semantics of PIP action } \\
\text { message structure }\end{array}$ & $\begin{array}{l}\text { Combination with other technologies } \\
\text { (e.g., semantic Web services) }\end{array}$ \\
\hline \multirow[t]{2}{*}{ papiNet } & \multirow[t]{2}{*}{ Messaging, dictionary } & Vocabulary & \multirow[t]{3}{*}{ TPA in Word format } & \multirow[t]{3}{*}{$\begin{array}{l}\text { Automate the setting of collaboration } \\
\text { agreements. }\end{array}$} \\
\hline & & Set of business documents & & \\
\hline Chem e - Standards & Messaging; BP & $\begin{array}{l}\text { Dictionary } \\
\text { CIDX documents }\end{array}$ & & \\
\hline RAPID, AgXML & $\begin{array}{l}\text { RAPID: EDI X12; XML; Bar coding; AgXML: } \\
\text { messaging; BPs }\end{array}$ & Dictionary & & Needs further development. \\
\hline PIDX & Messaging; BP; dictionary & Petroleum industry data dictionary & Semantic conflicts & $\begin{array}{l}\text { Needs further development (still in incipient } \\
\text { phase). Automated tools to solve semantic } \\
\text { conflicts (e.g., different units of measure } \\
\text { and attributes) }\end{array}$ \\
\hline$J A D M$ & & Common Vocabulary & & $\begin{array}{l}\text { Needs further development } \\
\text { (still in incipient phase). }\end{array}$ \\
\hline \multirow[t]{2}{*}{ TexWave } & \multirow[t]{2}{*}{ Messaging } & XML template for business documents & $\begin{array}{l}\text { High diversity of internal } \\
\text { applications used by } \\
\text { community members }\end{array}$ & $\begin{array}{l}\text { Automate the setting of collaboration } \\
\text { agreements. }\end{array}$ \\
\hline & & Dictionary & & \\
\hline \multirow[t]{2}{*}{ CEC-made-shoe } & \multirow[t]{2}{*}{ Messaging; BPs } & \multirow[t]{2}{*}{$\begin{array}{l}\text { Common set of XML-based business } \\
\text { documents }\end{array}$} & $\begin{array}{l}\text { No support to automatically } \\
\text { establish collaboration agreements. }\end{array}$ & Early development stage. \\
\hline & & & $\begin{array}{l}\text { Messaging platform not } \\
\text { compliant with ebXML Messaging } \\
\text { Specifications. }\end{array}$ & $\begin{array}{l}\text { Requires further development, addressing } \\
\text { cross-industry communication. }\end{array}$ \\
\hline
\end{tabular}


Table 5

Major initiatives for the footwear industry: an overview

\begin{tabular}{|c|c|c|c|c|}
\hline Initiative & Main outcomes & Layers & Strengths & Weaknesses \\
\hline EFNET & $\begin{array}{l}\text { - Identification of the main e-commerce } \\
\text { initiatives }\end{array}$ & (1) $\mathrm{M}$ & & \\
\hline SHOENET & $\begin{array}{l}\text { - SHOENET messaging platform; } \\
\text { - } 17 \text { XML-based BDocs }\end{array}$ & (1) $\mathrm{M}$ & $\begin{array}{l}\text { - Set of common BDocs ensuring } \\
\text { semantic interoperability }\end{array}$ & $\begin{array}{l}\text { - Proprietary messaging platform } \\
\text { not compliant with ebXML } \\
\text { Messaging Specifications }\end{array}$ \\
\hline CEC-made-shoe & $\begin{array}{l}\text { - Operational infrastructure for the } \\
\text { footwear industry } \\
\text { - Identification of the main interoperability } \\
\text { requirements in the footwear sector }\end{array}$ & $\begin{array}{l}\text { (1) } \mathrm{M} \\
\text { (2) } \mathrm{BP}\end{array}$ & $\begin{array}{l}\text { - Integration between messaging and } \\
\text { process-oriented platforms }\end{array}$ & $\begin{array}{l}\text { - Proprietary messaging platform } \\
\text { not compliant with ebXML } \\
\text { Messaging Specifications } \\
\text { - Needs further work to integrate } \\
\text { the two operational infrastructures }\end{array}$ \\
\hline
\end{tabular}

Note: $\mathrm{M}$ - messaging; BP - business process; BDocs - business documents.

and integration. It is based on workflow technology and allows SMEs to design, control and monitor their intra- and inter-BP workflows. BPs' choreography and BDocs exchanged are globally specified and stored in a repository. PICShoe system supports the specification of individual and concrete workflow processes, which will be enacted locally in each partner's environment. It knows the activities to be performed, their sequence in the BP. It differentiates human-based from automatic activities, and coordinates their assignment and execution. When a certain activity is assigned to a certain employee, a ToDoList is made available by PICShoe system, which allows users in the company to ask for an activity and execute it. The system will activate a business function that is encapsulated by an activity by opening a window in the user's computer, showing the information received from the previous activity. In a complex scenario (e.g., where some activities involve both employees and interactions with the company's IT system), the PICShoe system enables the realization of the read/write functionality of information to/from existing systems [6].

Fig. 5 illustrates an example of Order BP involving both human and automatic activities, using the PicShoe system.

Fig. 6 illustrates the transactions/flow of BDocs exchanged between a buyer (e.g., Producer) and a seller (e.g., Supplier).

An Order Dispatcher system has been implemented for an Order Dispatcher BP, based on a distributed workflow management system. This BP involves an Order Dispatcher (OD), a Producer (P) and a Supplier (SppC). Fig. 7 illustrates the conceptual design (Fig. 7a) and the workflow design (Fig. 7b) of the process executed by P. In this Order Dispatcher system, the Order Dispatch BP is organized as three separate sub-processes. The three top-level activities are executed by the OD entity. Firstly, the OD validates and dispatches an order. Next, the order is split in its items, and for each one an automatic activity is executed. Each multi-instance activity triggers the instantiation of a separate workflow process.

The Handle Supplier Order is an automatic activity aimed at instantiating the sub-process to be executed by SppC in order to provide the components required to build the shoe, which have been specified in the order. Additionally, a data structure is passed from activity to activity. In this way, the system presents context information to the entity executing a certain activity.

\section{Conclusions and future research work}

Economic factors, market conditions, and the development and adoption of Internet-based technologies have determined small and medium size enterprises (SMEs) to adopt new ways of undertaking business. As consequence, new forms of collaboration have emerged, such as CNs [1].

Despite the high number of tools and infrastructures supporting (or claming to support) seamless interoperability in a collaborative business environment, seamless connectivity is still unachieved $[3,4,58]$.

Significantly, we note the increasing interest in developing independent and industry-specific standards, aiming at supporting seamless interoperability for multiple participants with different but symbiotic goals, leading to an evolving business environment, rather than a traditional economic model. This trend is intrinsically collaborative and pictures the characteristics of the current networked business arena.

By tracking the research pursued so far in the area of semantic interoperability, we note that sharing services and content, expertise in certain activities or BPs, dictionaries, vocabularies, or business documents (BDocs) is (and will continue to be) part of the collaborative behaviour in a collaborative networked environment (CNE). However, these approaches are in an incipient phase, and deal with incomplete, inconsistent and dynamic knowledge and content, and (many times) unsolved data and meta-data privacy issues, or digital rights management.

We believe industry-specific initiatives bring a challenging and promising solution towards achieving seamless interoperability in business networks. However, most of these initiatives are at their infancy, requiring further developments, and the big challenge will be to achieve cross-industry seamless interoperability.

Table 3 contains a brief overview of major independent and industry-specific initiatives, presenting their aim, supporting organizations, and versioning. Table 4 presents their main elements, major drawbacks towards attaining seamless interoperability, and possible solutions to overcome these limitations.

Each of the initiatives presented tackles semantic interoperability differently, e.g., semantic interoperability in ebXML relays on Core Competences, while in the vertical initiatives it relies on industry-specific dictionaries or vocabularies (e.g., RosettaNet) or a common set of business documents. Development efforts are being pursued to extend their semantic capabilities (e.g., [35] for ebXML; [37] for RosettaNet).

Few studies comparing independent and industry-specific initiatives are available. Related to B2B standards, an analytical approach comparing ebXML and RosettaNet is available in [59]. A comparison of XML-based B2B integration frameworks is presented in [60]. Other study on frameworks for e-Business is available in [19]. However, these studies are more descriptive than comparative and many issues are not valid anymore.

Relevant e-business frameworks are analyzed in [36], indicating their site initiator and purpose. The study also compares eight frameworks: BPML, cXML, ebXML, OAGIS, papiNet, RosettaNet, $\mathrm{xCBL}$ and XPDL, having BDocs, BPs and messaging issues as basis for comparison. The properties and standardization of 12 XMLbased e-business frameworks is available in [60]: ebXML, BPEL, BPML, CIDX, cXML, OAGIS, papiNet, PIDX, RosettaNet, UBL, XCBL 
and XPDL. The analysis emphasizes their commonalities, differences and regularities concerning BPs, BDocs, and messaging.

All the initiatives addressed in this article represent a step forward towards seamless interoperability in a CNE. However, there are many challenges to explore: of technical nature, theoretical foundations (e.g., scalability), operational (e.g., gathering and maintaining the semantic content in domain communities, lack of appropriate supporting tools), economic (e.g., the added value of semantics when the return of investment may come downstream, performance assessment, for example, to support a join/leave/remain decision [8]), social (e.g., interplay among domain communities) and political (e.g., legal, security and privacy implications). All these directions require urgent further development.

We also suggest the consideration of formal/mathematical approaches (e.g., category theory, Godement calculus, natural transformations). And, as indicated by [54] the engagement of other theories or communities (e.g., social networks) to analyze semantic interoperability approaches in CNs, taking advantage of the symbiosis between social networks and CNs. Further developments should also focus on automated solutions to resolve incompatible business and data vocabularies for the above mentioned technologies (e.g., currently these problems are solved by custom code). Future research should also focus on assessing the benefits attained by adopting an industry-specific standard.

All vertical initiatives previously mentioned aim at achieving seamless interoperability within a business community by making use of emergent technologies. However, the approach is different (e.g., PIDX develops and maintains both EDI and XML implementation guidelines, while RosettaNet focuses only on XML technologies). There are, thus, some common points: most industryspecific initiatives use a two-layered approach: messaging and BPs. CEC-made-shoe approach is in line with these developments: it has a two-layered infrastructure, and makes use of a common set of XML-based business documents especially developed for this sector.

Table 5 briefly summarizes major initiatives targeting the footwear industry and their main outcomes.

EFNET and SHOENET projects contain only a single layer: the messaging layer, which does not answer SMEs' current needs in this sector. While EFNET and SHOENET projects refer to supply chain concept for the footwear industry, CEC-made-shoe EU Integrated Project introduces the concept of collaborative networked environment, which overcomes its constraints allowing adaptation of shoe product chain to dynamic market demands. The way in which these projects have evolved illustrates the trend of adopting emerging Internet-based technologies.

CEC-made-shoe project is the only approach that tackles BP issues by integrating two layers: a secure messaging infrastructure, and a BP-oriented platform, which makes use of workflow technologies for BP coordination and choreography. Therefore CEC-made-shoe project is the most advanced development towards deploying an interoperable collaborative business environment for the footwear industry, and uses outcomes of previous research projects (e.g., the 17 XML-based Business Documents, SHOENET messaging platform). Compared to ebXML, the BP layer developed within the scope of CEC-made-shoe Integrated Project represents a compression of ebXML's Business Process and Collaboration Protocol Profile/Agreement layers. Its main drawback, however, is the use of a proprietary messaging platform not compliant with ebXML Messaging Specifications. Additionally this approach does not tackle business/economic interoperability issues. Real data will be collected form SMEs in the footwear sector to assess the benefits obtained by using this operational infrastructure.
The feasibility to use an open-source based messaging platform for the footwear sector is currently being analyzed in order to avoid any proprietary lock-in. Further work will also be pursued to integrate the two operational infrastructures: for up-stream and down-stream segments. Real tests from industry will also be elaborated.

\section{Acknowledgements}

The first author acknowledges FCT for PhD grant BD/SFRH/ $19751 / 2004$. The authors would like to thank the anonymous reviewers for their valuable suggestions, which have enhanced the quality of the article.

\section{References}

[1] C.-M. Chituc, A. Azevedo, Towards a self-forming business networking environment, Proceedings 7th International Conference on Enterprise Integration Systems-ICEIS, 2005, pp. 455-548, ISSN 9728865198.

[2] F. Nachira, Towards a network of digital business ecosystems fostering the local development, 2002 (discussion paper retrieved from http://europa.eu.int/information_society/topics/ebusiness on September 20, 2006).

[3] L. Camarinha-Matos, H. Afsarmanesh, Elements of a base VE infrastructure, Computers in Industry 51 (2003) 139-163.

[4] J.T. Pollock, The biggest issue: interoperability vs. integration, eAI Journal (2001) 48-52.

[5] C.-M. Chituc, C. Toscano, A.L. Azevedo, Towards the creation of a digital business ecosystem for the shoe manufacturing domain, in: Proc. IEEE DEST, 2007, 88-93.

[6] C.-M. Chituc, C. Toscano, A.L. Azevedo, Interoperability in Collaborative Networks: an innovative approach for the shoe up-stream segment, in: R. Goncalves, et al. (Eds.), Enterprise Interoperability II-New Challenges and Approaches, Springer London, 2007, pp. 557-568.

[7] C.-M. Chituc, A. Azevedo, Business networking-the technological infrastructure support, in: M. Cunha, G. Putnik (Eds.), Knowledge and Technology Management in Virtual Organizations, Idea Publishing/IGI Global, 2006, pp. 334-354, Chapter 16.

[8] C.-M. Chituc, S.Y. Nof, The Join/Leave/Remain (JLR) decision problem in collaborative networked organizations, Computers \& Industrial Engineering 53 (2007) 173-195.

[9] R. Camacho, D. Guerra, N. Galeano, A. Molina, An integrative approach for Vo planning and launching, in: L. Camarinha-Matos, H. Afsarmanesh, A. Ortiz (Eds.) Collaborative Networks and their Breeding Environments, Springer, 2005.

[10] G. Jiménez, N. Galeano, T. Nájera, J.M. Aguirre, C. Rodriguez, A. Molina, Methodology for business model definition of collaborative networked organizations, in: L. Camarinha-Matos, H. Afsarmanesh, A. Ortiz (Eds.), In Collaborative Networks and their Breeding Environments, Springer, 2005, pp. 347-354.

[11] S. Alexakis, B. Kolmel, T. Heep, VO in Industry: State of the Art. In Collaborative Networked Organizations: A Research Agenda for Emerging Business Models, Kluwer, 2004

[12] D.J. Lewis, Partnership for Profit: Structuring and managing Strategic Alliances, The Free Press, New York, 1990.

[13] A. Parcker, Inter-firm collaboration and the new product development process, Industrial Management and data Systems 100 (6) (2000).

[14] J.A. Holton, Building trust and collaboration in a virtual team, Team Performance Management: An International Journal 7 (3-4) (2001) 36-47.

[15] T.M. McCarthy, S.L. Golicic, Implementing collaborative forecasting to improve supply chain performance, International Journal of Physical Distribution and Logistic Management 32 (6) (2002) 431-454.

[16] C.M. Chituc, A. Azevedo, Multi-perspective challenges on collaborative networks business environment, in: L. Camarinha-Matos, H. Afsarmanesh, A. Ortiz (Eds.), Collaborative Networks and Breeding Environment, Springer, 2005, pp. 25-32, ISBN 978-0387-28259-6.

[17] IEEE, IEEE Standard Computer Dictionary: A Compilation of IEEE Standard Computer Glossaries, Institute of Electrical and Electronics Engineers, NY, 1990.

[18] D. Chen, G. Doumeingts, Basic concepts and approaches to develop interoperability of enterprise applications, in: L. Camarinha-Matos, H. Afsarmanesh (Eds.), Processes and Foundations for Virtal Organizations, Kluwer, 2004, pp. 323-330 ISBN 1-4020-7638.

[19] H. Li, XML and industrial standards for electronic commerce, Knowledge and Information Systems 2 (4) (2000) 487-497.

[20] A. Sheth, C. Ramakrishan, C. Thomas, Semantics for the semantic web: the implicit, the formal, and the powerful, International Journal on Semantic Web \& Information Systems 1 (1 (Jan-March)) (2005) 1-18.

[21] M. Klein, Combining and relating ontologies: an analysis of problems and solutions, Workshop on Ontologies and Information Sharing IJCA, USA, 2001.

[22] IDEAS Consortium, Gap analysis and required activities in research technology and standardization to close the gap, Deliv. D3.1 and D3.2, 2002.

[23] Y. Ducq, D. Chen, B. Vallespir, Interoperability in enterprise modelling: requirements and roadmap, Advanced Engineering Informatics 18 (2004) 193-203. 
[24] F. Verenadat, Enterprise Modeling and Integration-Principles and Applications, Chapman \& Hall, 1996 ISBN 0-412-605503.

[25] C.F. Crargil, Information Technology Standardization: Theory, Process, and Organization, Digital Press, Bedford, 1989.

[26] H. Li, T.J. Williams, The interconnected chain of enterprises as presented by the Purdue Enterprise Reference Architecture, Computers in Industry 42 (2000) 265274.

[27] H. Li, T.J. Williams, Interface design for the Purdue enterprise reference architecture (PERA) and methodology in e-Work, Production Planning \& Control 14 (8) (2003) 704-719.

[28] IFAC/IFIP, GERAM: Generalized Enterprise Reference Architecture and Methodology, IFAC/IFIP Task Force on Architectures for Enterprise Integration, 2000.

[29] J.A. Zachman, A Framework for Information Systems Architecture, IBM Systems Journal 26 (3) (1987).

[30] Workflow Management Coalition, The Workflow Management Coalition Specifications: Terminology and Glossary, 1999 (http://www.wfmc.org)

[31] C. Bussler, B2B Integration-Concepts and Architectures, Springer, 2003.

[32] SWWS-Semantic Web Enabled Web Services: Analysis of B2B Standards and Systems, Deliv. D1.1, 2003.

[33] M.L. Nelson, M.J. Shaw, The adoption and diffusion of interorganizational system standards and process innovations, MISQ Special Issue Workshop-Standard Making: A Critical Frontier for Information Systems, 2005.

[34] M.L. Nelson, M.J. Shaw, W. Qualls, Interorganizational system standards development in vertical industries, Electronic Markets 15 (4) (2005) 378-392.

[35] B. Hofreiter, C. Huemer, B2B integration: aligning ebXML and ontology approaches, in: Proc. EURASIA-ICT Conference, 2002.

[36] J.M. Nurmilaakso, P. Kotinurmi, A review of XML-based supply-chain integration, Production Planning \& Control 15 (6 (September)) (2004) 608-621.

[37] P. Katinurmi, T. Vitvar, Adding semantics to RosettaNet specifications, in: 15th International World wide Web Conference, 2006 (poster).

[38] M. Uschold, Where are the Semantics in the Semantic web? Artificial Intelligence Magazine 24 (3) (2003) 25-36.

[39] H. Zhuge, Semantic Grid: Scientific Issues, Infrastructure, and Methodology, Communications of ACM 48 (4) (2005).

[40] P.R.S. Visser, D.M. Jones, T.J.M. Bench-Capon, M.J.R. Shave, Assessing heterogeneity by classifying ontology mismatches, in: Proc. Int. Conf. on Formal Ontology in Information Systems (FOIS), IOS Press, 1998, pp. 148-162.

[41] T.R. Gruber, A translation approach to portable ontology specifications, Knowledge Acquisition 5 (1993) 199-220.

[42] M. Cristani, R. Cuel, A survey on ontology creation methodologies, International Journal on Semantic Web \& Information Systems 1 (2) (2005) 49-69.

[43] M. Kavouras, A unified ontological framework for semantic integration, in: Proc Int. Workshop on Next generation Geospatial Information, 2003, 19-21.

[44] H. Wache, T. Vogele, U. Visser, U. Stuckenschmidt, G. Schuster, H. Neumann, S Hubner, Ontology-based integration of information-a survey of existing approaches, in: H. Stuckenschmidt (Ed.), Proc. Workshop: Ontologies and Information Sharing, 2001, 108-117.

[45] A.J. Duineveld, R. Stoter, M. Weiden, B. Kenepa, V.R. Benjamins, Wondertools, A comparative study of ontological engineering tools, International Journal of Human-Computer Studies 6 (52) (2000) 111-1133.

[46] D. Fensel, I. Horrocks, Harmelen, F. van, D. McGuiness, D. Patel-Schneider, OIL: ontology infrastructure to enable the semantic web, IEEE Intelligent System 16 (2) (2001).

[47] M. Gruninger, Using model-theoretic invariants for semantic interoperability (presentation retrieved on April 10, 2006, from: http://www.nist.gov/psl).

[48] C. Bock, M. Gruninger, PSL: a semantic domain for flow models, Software System Models 4 (2005) 209-231.

[49] A.F. Cutting-Decelle, L. Pouchard, J.J. Michel, R. Young, B. Das, Using standards based approaches to information sharing and interoperability in manufacturing decision support, in: Proc. Conf. Flexible Automation \& Intelligent Manufacturing Conference, 2004.

[50] L. Pouchard, N. Iveciz, C. Schienoff, Ontology engineering for distributed collaboration in manufacturing, in: Proc. AIS Conference, 2000

[51] J.T. Pollock, R. Hodgson, Adaptive Information: Improving Business through semantic Interoperability, Grid Computing, and Enterprise Integration, WileyInterscience, 2004

[52] D. Roure, N.R. Jennings, N. Shadbolt, The semantic grid: past, present and future Proc. IEEE 93 (3) (2004) 669-681.

[53] M. Uschold, M. Guninger, Ontologies and semantics for seamless connectivity, SIGMOD Record 33 (4) (2004).
[54] D.De Roure, J. Frey, D. Michaelides, K. Page, The collaborative semantic grid, in: Proc. CTS, 2006

[55] C. Toscano, C.-M. Chituc, et al. Business Model of Strategic Collaborative Network, Deliv. D4.2.1 CEC-made-shoe EU Integrated Project, INESC Porto, 2005.

[56] European Commission, ICT and e-business in the footwear industry, Sector impact study no. 2, 2006 (retrieved from http://www.ebusiness-watch.org on January 12, 2007).

[57] C. Toscano, High Level Design of Integration Platform of SCN, Deliverable D4.2.2, CEC-made-shoe EU Integrated Project, INESC Porto, 2005.

[58] R. Jardim-Goncalves, A. Grilo, A. Steiger-Garcao, Challenging the interoperability between computers in industry with MDA and SOA, Computers in Industry 57 (2006) 679-689.

[59] M. Pusnik, M.B. Juric, I. Rozman, B. Sumak, A Comparison of ebXML and RosettaNet, 2000 (white paper).

[60] J.-M. Nurmilaakso, P. Kotinurmi, H. Laesvouri, XML-based e-business frameworks and standardization, Computer Standards \& Interfaces 28 (2006) 585-599.

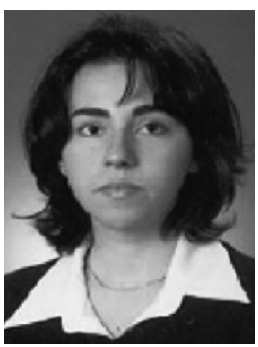

Claudia-Melania Chituc is $\mathrm{PhD}$ candidate at the Faculty of Engineering of the University of Porto, Portugal (Electrical and Computer Engineering Department), and researcher at INESC Porto. She received a MSc degree from the same university in June 2004, in the area of information technology for enterprise management. Claudia-Melania has pursued research in Portugal, USA (Purdue University, School of Industrial Engineering) and Romania. Her education is inter-disciplinary: she is both computer engineer and economist. She graduated Politehnica University of Bucharest, Romania (computer science and engineering) in 2002, and the Academy of Economic Studies, Bucharest, in 2000 (being specialized in Finances, Insurances, Banks and Stock Exchange). Claudia-Melania's current areas of research include: interoperability and performance assessment in collaborative networked organizations, and business process management.

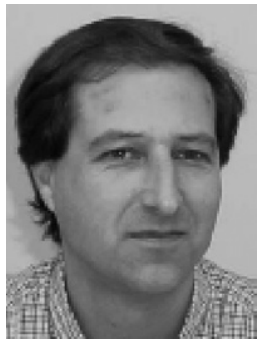

César Toscano has a degree of electrical engineering from the Engineering Faculty of Porto University (1985). Currently, he is senior technician in the "Manufacturing Systems Engineering Unit" of INESC PORTO. Among previous positions, he was Assistant in the "Instituto Superior e Politécnico de Gaia" (19862000) and scientific associate in "CERN, Conseil Européen pour la Recherche Nucléaire" (1988). He participated in several R\&D European projects: "Cecmade-shoe: Custom, Environment and Comfort made shoe", "B-MAN: Business Mobile Agent Network", "MyFashion.eu: Added Value for the Consumer Through Integrated, Extended Fashion Products", "Co-OPERATE: Co-operation in Dynamic Networked Organisations" and "X-CITTIC: A Planning and Control System for Semiconductor Virtual Enterprises". He has interests in information systems development, systems architecture, distributed systems, enterprise integration, enterprise modelling and supply chain management.

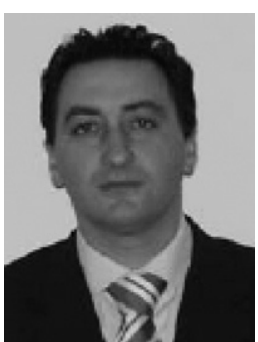

Américo Azevedo has a $\mathrm{PhD}$ in the area of advanced planning systems for networking enterprises. Since 1998, he has been teaching electrical and computer engineering in the School of Engineering of the University of Porto, Portugal, and since 1995 he has been a researcher and project leader in INESC PORTO. He is author of many articles in international journals and technical publications, and he has been active in preparing and participating in $R \& D$ projects involving industrial companies. His main research interests are in the collaborative business networking, business process management, and operations management. 